\title{
Sex differences in cortisol and memory following acute social stress
}

$8 \quad{ }^{a}$ Neuropsychology and Cognitive Health Program, Baycrest, Toronto, ON, Canada.

$9 \quad$ b Psychology Department, University of Toronto, Toronto, ON, Canada

$10{ }^{\mathrm{c}}$ Djavad Mowafaghian Centre for Brain Health, Department of Psychology, University of British

11 Columbia, Vancouver, BC, Canada.

$12{ }^{\mathrm{d}}$ Psychology Department, Western University, London, ON, Canada.

13 * corresponding author; Email: liisa.galea@ubc.ca

\section{ORCiD IDs}

16 KJM: 0000-0002-3827-5814

17 TEH: 0000-0002-5993-2633

18 PASS: 0000-0001-7432-1446

19 AKT: 0000-0001-5660-4127

20 EH: 0000-0003-4487-9013

21 LAMG: 0000-0003-2874-9972

22

23

24 Abstract: 300 words

25 Manuscript: 8746 words 


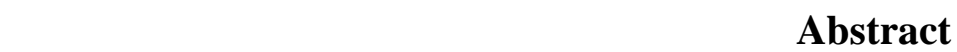

Objective: Older adults with amnestic mild cognitive impairment (aMCI) develop Alzheimer's type dementia approximately ten times faster annually than the normal population. Adrenal hormones are associated with aging and cognition. We investigated the relationship between acute stress, cortisol, and memory function in aMCI with an exploratory analysis of sex. Method: Salivary cortisol was sampled diurnally and during two test sessions, one session with the Trier Social Stress Test (TSST), to explore differences in the relationship between cortisol and memory function in age-normal cognition (NA) and aMCI. Participants with aMCI ( $\mathrm{n}=6$ women, 9 men; mean age=75) or similarly aged NA ( $\mathrm{n}=9$ women, 7 men, mean age=75) were given tests of episodic, associative, and spatial working memory with a psychosocial stressor (TSST) in the second session. Results: The aMCI group performed worse on the memory tests than NA as expected, and males with aMCI had elevated cortisol levels on test days. Immediate episodic memory was enhanced by social stress in NA but not in the aMCI group, indicating that stress-induced alterations in memory are different in individuals with aMCI. High cortisol was associated with impaired performance on episodic memory in aMCI males only. Cortisol in Session 1 moderated the relationship with spatial working memory, whereby higher cortisol was associated with worse performance in NA, but better spatial working memory in aMCI. In addition, effects of aMCI on perceived anxiety in response to stress exposure were moderated by stressinduced cortisol in a sex-specific manner. Conclusions: We show effects of aMCI on Test Session cortisol levels and effects on perceived anxiety, and stress-induced impairments in memory in males with aMCI in our exploratory sample. Future studies should explore sex as a biological variable as our findings suggests that effects at the confluence of aMCI and stress can be obfuscated without sex as a consideration. 


\section{Introduction}

Normal aging results in declines in some cognitive domains, such as episodic memory, but not in others, such as experiential knowledge (Grady, 2012). Cognitive decline with aging is correlated with region-specific changes in prefrontal cortex and medial temporal lobe, a key change being hippocampal volume loss (Raz \& Rodrigue, 2006) and these declines are accelerated in those with suspected Alzheimer's Disease (AD) (Shi et al., 2009). Older adults with mild cognitive impairment (MCI) develop clinical dementia of the AD type at a rate of $10-30 \%$ annually, depending on MCI subtype, whereas those without MCI develop dementia at a rate of only 1\% to 2\% annually (Busse et al., 2003; Lupien et al., 1998). Thus, it is critical to identify neurobiological factors that may distinguish MCI from normal aging, such as differences in cortisol levels and their response to stress. Dysregulation of the hypothalamic-pituitary-adrenal (HPA) system, including the stress hormone cortisol, has been linked to memory performance, aging, and hippocampal volume (Lupien et al., 1998; Sindi et al, 2014; Justice, 2018). Indeed, participants with suspected AD have higher levels of plasma (morning, $24 \mathrm{~h}$ release) or morning cerebrospinal fluid (CSF) cortisol than those experiencing normal cognitive aging (Hartmann et al., 1997; Doecke et al., 2012; Laske et al., 2011). Morning (CSF) levels of cortisol are also higher in MCI due to suspected AD, referred to as amnestic MCI (aMCI), as compared to older adults experiencing normal aging (NA) or MCI of other types (Popp et al., 2015). Moreover, aMCI individuals with higher morning CSF cortisol levels experienced accelerated clinical worsening and cognitive decline than those with a lower levels of cortisol, with a higher proportion of males in the aMCI group (Popp et al., 2015). Intriguingly, despite higher levels of cortisol, MCI participants experience lower levels of perceived stress during cognitive performance compared to agematched healthy controls (Guerdoux-Ninot \& Trouillet, 2019). Previous studies have rarely examined multiple timepoints of cortisol, stress induced cortisol, diurnal cortisol, or have used biological sex as a discovery variable, all factors which may contribute to the findings (Hidalgo et al., 2019; Yan et al., 2018). Cortisol is well known to vary in a diurnal pattern (Adam et al., 2017) and diurnal patterns are flattened in dementia of the AD type (Rasmuson et al., 2011; Ferrari et al., 2001). Importantly, to our knowledge, stress-induced cortisol has not been studied in relation to aMCI status and memory performance. Acute stress may be a more salient variable of HPA dysregulation to examine whether it can perturbate memory. Indeed, normally aging older adults who show less cortisol reactivity to acute stress using the Trier Social Stress Test (TSST) are more at risk to develop cognitive decline characteristic of MCI within 5 years (de Souza-Talarico et al., 2020). Thus, it is important to examine not only diurnal fluctuations in cortisol but also response to acute stress to determine whether these 
85

86

87

88

89

90

91

92

93

94

95

96

97

98

99

100

101

102

103

104

105

106

107

108

109

110

111

112

113

114

115

116

biomarkers modulate memory and how they may relate to progression to neurodegenerative disease. In addition, perceived stress, along with stress-induced cortisol, may be as important to investigate (Aggarwal et al., 2014). Furthermore, sex differences must be considered given previous studies have identified sex differences in cortisol levels in response to stress (Kudielka \& Kirschbaum, 2005) which vary by age (for review see Hidalgo et al., 2019). Indeed, few studies to date have examined the relationships between cortisol, stress, memory, and potential differences between males and females in older populations (for review see Hidalgo et al., 2019), particularly with regards to MCI or AD.

Sex differences are seen in incidence of MCI (Gale et al., 2016; Koran et al., 2017; Mielke et al., 2014; Duarte-Guterman et al., 2020), with males more likely to develop MCI (both aMCI and nonamnestic subtypes) than females (Jack et al., 2019; Roberts et al., 2012), although there are conflicting reports that are likely due to methodological differences (e.g. Mielke et al., 2014). However, AD disproportionately affects females, with significant sex differences observed with regards to severity, neuropathological markers, and rates of cognitive decline (Irvine et al., 2012; Koran et al., 2017; Sohn et al., 2018). Sex differences in incidence of AD are not seen uniformly (Jack et al., 2019) and may depend on geographic location (Nebel et al., 2018) or greater longevity in women (Mielke et al., 2014). However, there are other sex differences in MCI to AD progression and symptom severity (Sundermann et al., 2017). For example, women tend to develop MCI at a later age, perhaps benefitting from their established superior verbal memory (Sundermann et al., 2017), but progress to AD more rapidly than men when adjusted for age (Irvine et al., 2012; Sohn et al., 2018). Sex differences in the trajectory of cognitive decline are also seen in MCI (Koran et al., 2017; Sohn et al., 2018). Indeed, females with more AD-associated neuropathology (total-tau and amyloid-beta [A $\square 42$ ] in cerebrospinal fluid), show greater declines in hippocampal volume and cognition compared to males, particularly among MCI individuals using the ADNI database (Koran et al., 2017; Sohn et al., 2018). Furthermore cognitive differences in verbal learning, delayed recall, visual learning and memory between NA and MCI females were significantly greater than those between NA and MCI males (Gale et al., 2016). This pattern in sex differences persisted in those with AD (Gale et al., 2016). Thus, sex differences in severity and progression to $\mathrm{AD}$ are seen in individuals with MCI and identifying the biological causes of this phenomenon is critical to treatment and prevention.

Gonadal production of sex steroids is reduced, but not entirely eliminated, with age in women and, to a lesser extent, men; however, adrenal cortisol production increases with age (Laughlin \& Barrett-Connor, 2000). Although both sexes show increased cortisol levels with increased age, this effect is 3 times more pronounced in females (Otte et al., 2005) and increased cortisol levels are linked 
117

118

119

120

121

122

123

124

125

126

127

128

129

130

131

132

133

134

135

136

137

138

139

140

141

142

143

144

145

146

147

148

to poorer cognition and smaller hippocampal volume in older age (Lupien et al., 1998). Furthermore, as mentioned above females with MCI and AD present with greater declines in hippocampal atrophy and cognition than males (Irvine et al., 2012; Koran et al., 2017; Sohn et al., 2018), highlighting that the underlying pathophysiology of AD may be different in men and women and should be further explored. Although sex differences in AD have been identified, studies are scarce and even more so in MCI groups.

In this study we explored the relationship between diurnal fluctuations in cortisol, stressinduced cortisol, and memory performance in older adults experiencing aMCI and NA. A spatial working memory task known to be reliant on the integrity of the prefrontal cortex (Courtney et al., 1998) and an episodic and associative memory task known to be reliant on hippocampal integrity (Eichenbaum, 2017) were selected based on the number of glucocorticoid receptors and therefore sensitivity to cortisol in these brain regions (Dedovic et al., 2009) and the potential of fluctuations in cortisol to influence cognitive efficiencies. Because little is known as to whether there are sex differences in the relationship between aMCI and cortisol, we also used exploratory analyses of sex effects in the present study. We hypothesised that stress-induced cortisol, via the application of a psychosocial stressor (Trier Social Stress Test; Kirschbaum et al., 1993), would worsen memory scores and possibly alter perceived anxiety in individuals with aMCI compared to NA and that there would be sex differences in these effects.

\section{Materials and methods}

\section{Participants}

Older adults with age-normal memory (normal cognitive aging- NA) and with mild memory decline (aMCI) suggestive of neurodegenerative disease of the Alzheimer type (Albert et al., 2011) were recruited for this study and provided informed voluntary consent to participate. The following brief battery of neuropsychological measures were administered during Session 1 to confirm group membership: cognitive screening (Mini-Mental Status Exam (MMSE, Folstein \& Folstein, 1974), expressive vocabulary (Vocabulary, Wechsler, 1997), attention tests involving auditory attention span (Digit Span, Wechsler, 1997) and speed and attention switching (Trail Making Tests A and B; -Spreen \& Strauss, 1998), confrontation naming (Boston Naming Test, Kaplan et al., 1983), visuospatial construction, and immediate memory (Rey-Osterrieth Complex Figure-copy and immediate recall, Spreen \& Strauss, 1998); and mood status (Hospital Anxiety and Depression Scale, HADS, Zigmond \& Snaith, 1983). Criteria for establishing aMCI status were adherent to those described in Petersen (2004) 
149

150

151

152

153

154

155

156

157

158

159

160

161

162

163

164

165

166

\section{7}

168

169

170

171

172

173

174

175

176

177

178

179

180

and Albert et al. (2011). Participants were classified with single domain amnestic MCI if memory performance was revealed to be the only cognitive domain among those tested (which included attention, psychomotor speed, memory, language, visual spatial ability, and executive function) for which age-scaled scores were lower than expected based on estimated verbal IQ (established on a test of expressive vocabulary) and based on demonstrated performance in the other cognitive domains examined (see Table 1).

\section{Normal Cognitive Aging (NA) group}

Fifteen older adults (age 61-86 years) experiencing NA were recruited via community talks, newspaper advertisements, and databases of research volunteers. Prior to invitation to participate, normal general cognitive status, using the Telephone Interview for Cognitive Status, and health status were confirmed in a telephone screening interview. At the first of two sessions, health history was further queried to verify that there was no history of a neurological, medical, or psychiatric disorder, substance abuse, or medications affecting cognition. As described, NA was confirmed during Session 1 by measuring performance on a brief battery of neuropsychological tests. Three participants initially recruited as NA were identified as meeting criteria for aMCI based on Session 1 interview and lower than age and education expected performance on immediate recall of a complex figure.

\section{Amnestic Mild Cognitive Impairment (aMCI) group}

Sixteen individuals (age 59-85 years), recruited from physician referrals, from databases of research volunteers, and from newspaper advertisement, were classified as meeting the National Institute on Aging-Alzheimer's Association classification criteria for aMCI (Albert et al., 2011). The single domain aMCI status of most of the aMCI participants had been previously established and the stability of this classification was confirmed by the interview and neuropsychological testing administered during Session 1. As previously stated, three of the participants initially recruited to the NA group were found to meet criteria for aMCI at Session 1 and were transferred to the aMCI group.

\section{[INSERT TABLE 1 HERE]}

\section{Procedure}

Participants completed alternate versions of episodic, associative, and spatial memory tasks across two test sessions (Figure 1) conducted 7-14 days apart. The TSST (Kirschbaum et al., 1993), a 
181 psychosocial stressor, was applied during the second test session and is described below. Salivary 182 cortisol was collected during both test sessions for all participants and diurnal samples were collected

183 as described below. Saliva was chosen because it most closely represents bioavailable cortisol (i.e., the 184 fraction of the circulating hormone that is biologically available to tissues).

Episodic memory

Two highly correlated versions (forms 5 and 6) of the Hopkins Verbal Learning Test-Revised (HVLT-R) were used (Session 1: form 6; Session 2: form 5; with the exception of one aMCI participant to whom they were presented in the opposite order). This task involves an oral presentation of a 12-item word list over three learning trials, followed by a 20-minute delayed recall trial and a forced choice yes/no recognition trial. The recognition trial consists of 24 items comprised of the 12 target words, six semantically related foils, and six un-related foils. Measures of interest included total

\section{Associative recognition}

A face-name associative recognition test, created by Troyer and colleagues (2011, 2012; images of faces (half male and half female) paired with aurally presented first names. Two versions of the task were used, each with 28 face-name pairs. During the task, 20 faces were individually presented on the computer screen for 6 seconds each with an inter-stimulus interval of 0.5 seconds; the examiner read the name associated with each face at the onset of each new face stimulus. Two study phases, differing only in stimulus presentation order, were administered in succession because our previous research indicated that item memory and association memory differences increase after repeated learning trials (Troyer et al., 2008). Only 16 of the 20 face-name pairs were considered test items as the first and last pairs in each study phase presentation were excluded to reduce primacy and recency effects on recognition accuracy. Following a 30 second delay, yes/no recognition testing was conducted with 24 face-name pairs presented, including eight intact pairs, eight recombined pairs, and eight new pairs presented in random order. During testing the examiner orally presented the name in the form of a question “Did I tell you this was [NAME]?” when the face appeared on the screen. Participants were instructed to say "yes” only to faces they had seen before that were paired with the correct name and "no" to faces they had not seen before, faces that were paired with the wrong name, or names they had not heard before. Immediately following testing, procedure verification was undertaken (i.e. 
213 participants retold the yes/no rules to the examiner). Participants were presented with unique, but

214 equivalent (Troyer et al., 2011), sets of face-name pairs during Sessions 1 and 2. Associative

215 recognition calculated as the difference between the proportion of correctly identified intact pairs (same 216 face-name pairs viewed at study) and proportion of false alarms to recombined pairs (different pairings 217 of previously viewed faces and names at study) was the primary measure of interest. The decision to 218 focus on associative recognition was based on our previous research demonstrating this measure was 219 most sensitive to aMCI and hippocampal volume loss (Troyer et al., 2012).

\section{Spatial working memory}

This task was modeled after the stimuli and procedures of Duff and Hampson (2001). A 4x5 rectangular array, measuring approximately $27 \mathrm{~cm}$ in length and $34 \mathrm{~cm}$ in width, consisting of coloured squares (10 colours, each represented twice) that were hidden under removable covers, was presented on a tabletop at which participants were seated. The coloured squares were randomly arranged on a uniform white backing and completely concealed beneath uniform white covers that could be temporarily lifted by participants to reveal the coloured square beneath. Participants were instructed to find all 10 pairs of matching coloured squares in as few choices as possible by lifting the covers two at a time. Prior to beginning the task, participants were familiarised with the colours of the test stimuli by having them view and name a set of 10 individual coloured squares. Each time a matching pair was located on the stimulus array, the examiner placed an individual coloured square representing the colour of the pair discovered at the top of the rectangular array, so participants did not need to remember which colour pairs had been found. Measures of interest included: the number of choices (squares uncovered) made in discovering all 10 matching pairs (criterion) and the time taken to reach criterion. Participants were told they would be timed and that they should attempt to locate all 10 pairs in as few choices as possible. Once they reached criterion, a second trial was immediately administered, with a third trial administered following a 30-minute delay. Locations of the coloured squares was constant within session but changed between Sessions 1 and 2 .

\section{Psychosocial stressor (TSST)}

The psychosocial stressor used in this study was modeled after the TSST (Kirschbaum et al.,

242 1993). The stressor was introduced immediately following the first saliva collection at 10:10h.

243 Participants were instructed to prepare a five-minute speech on the topic of 'The effect of tuna fishing 244 on the dolphins and other ocean animals' to be presented to a panel of three evaluators, including the 
245

examiner. They were given a pencil and paper and told to write down the points they would like to make in their speech for which they would have 10-minutes to prepare. The examiner then left the room and returned 10 minutes later, collected a saliva sample from the participant (anticipation period), and then led the participant to a conference room to give their speech. Participants were instructed to leave their written notes behind, to give their speech from memory, and to try and speak for five minutes, which was timed by the examiner. Immediately following the public speech, participants engaged in a five-minute serial subtraction task in which they were asked to count backwards aloud by 13 from the number 1022 as quickly and accurately as possible in front of the panel of evaluators. When an error was committed the participant was instructed to begin again from the number 1022. Because perceived stress/anxiety has been found to be lower in aMCI compared to NA individuals performing an attention-based task (Stroop-task; Guerdoux-Ninot \& Trouillet, 2019), participants were asked if they found the test anxiety provoking and if so to rate their perceived anxiety during the public speech from 0 (low anxiety) to 100 (high anxiety). Lastly, participants were led back to the test room to undertake memory testing (episodic, associative, spatial) and to provide additional saliva samples as described below.

\section{[INSERT FIGURE 1 HERE]}

\section{Saliva collection and analysis}

Multiple salivary cortisol samples were taken using the Salivette ${ }^{\mathrm{TM}}$ method (Sarstedt Inc, Sarstedtstraße, Numbrecht, Germany) across both the test sessions (for all participants) and at home (to examine diurnal variations). Participants were instructed not to eat, drink, or smoke for at least 30 minutes prior to saliva collection and to rinse their mouths with water 5 minutes before collecting the saliva. For the test sessions, both sessions were conducted in the morning from 10:00h to 12:00h. Saliva was collected at 10:10h and 12:00h at the first test session and during the second session at 10:10h; immediately following the anticipation period to the application of a psychosocial stressor ( 10:30h); 30 minutes following the application of the psychosocial stressor ( 11:00h); and at about 12:00h at the conclusion of the second test session. In addition, basal salivary diurnal cortisol samples were requested from participants across three agreed upon days intervening between the test sessions. Five samples were collected per day on the following schedule: 30-minutes after awakening (ranged from 5:30 am to 8:30h), 09:00, 16:00, 19:00, and 21:00h. Phone call reminders and verifications were provided by the examiner for each of the 4 specified clock times on all three collection days. 
277 Participants were given pre-labeled saliva collection tubes at the end of Session 1 and instructed to 278 store their collected samples in the home refrigerator. Collection time of day was further verified by 279 requiring participants to record the collection time on a label provided on the collection tube. The basal 280 samples were gathered from participants when they returned for the second test session.

281 Saliva was centrifuged at $1500 \mathrm{~g}$ and kept frozen at $-20^{\circ} \mathrm{C}$ prior to analysis. Cotton-based 282 collection is suitable for cortisol determinations (Büttler et al., 2018). Salivary cortisol was analyzed in 283 duplicate by the Neuroendocrinology Assay Laboratory at the University of Western Ontario (EH). An 284 established ${ }^{125}$ I solid-phase radioimmunoassay was used (Norman et al., 2010), based on antibody and 285 tracer obtained from Siemans Healthcare Diagnostics (Deerfield, IL). The Laboratory specialises in 286 saliva determinations. Briefly, saliva was analyzed directly, without extraction, using a $200 \mu \mathrm{L}$ aliquot 287 and an extended 3hr incubation at room temperature. The calibration curve was diluted 1:10 and ranged 288 from 0-138 nmol/L. The intra-assay coefficient of variation calculated across low, medium, and high 289 pools averaged $4.2 \%$ and the sensitivity of the assay was $<0.25 \mathrm{nmol}$ across 3 assay runs. All samples 290 from a given participant were analyzed in the same assay run and the average salivary cortisol concentration across the two duplicates (in nmol/L) was the value used for all statistical analyses. All cortisol data was log-transformed $\left(\log _{10}\right)$ due to non-normality. formulas for AUC one with respect to the ground (AUCg) and one with respect to the increase (AUCi) (Pruessner et al., 2003) using the following formulas:

$$
\begin{aligned}
A U C g & =\sum_{i=1}^{n-1} \frac{\left(m_{(i+1)}+m_{i}\right) * t_{i}}{2} \\
A U C_{i} & =A U C_{g}-m_{1} * \sum_{i=1}^{n-1} t_{i}
\end{aligned}
$$

Where $t_{i}$ - is the distance between time measurements), $m_{1}$ - is the first cortisol measurement

\section{Data Analyses}


307

308

309

310

311

312

313

314

315

316

317

318

319

320

321

322

323

324

325

326

327

328

329

330

331

332

333

334

335

336

337

338

using group (NA, aMCI) and sex (male, female) as the between-subject factors and with either Session $(1,2)$ or time of day (30 min, 9 AM, 4PM, 7pm, 9PM) or time during test Session $(10: 10,10: 30,11$, 1130) as the within-subjects factor. On certain measures (represented in Table 1) general linear model ANCOVA were conducted using group (NA, aMCI) as the between-subject factor. Post hoc analyses used Newman-Keul's comparisons. Due to the small sample size, exploratory analyses on possible sex effects were run using a Bonferroni correction on a priori analyses, using a two-tailed significance criterion of $p=0.10$. Otherwise statistical significance was $p=0.05$ was used for all other statistical tests conducted. Chi-square analyses were completed for frequency of sexes between groups and for anxiety scores. For exploratory moderation analyses hierarchical linear regressions were performed to test the overarching hypothesis that the magnitude of Session 1 and Session 2 cortisol moderates the relationship between group (MCI, NA) and perceived anxiety during the TSST, episodic memory, associative memory, or working memory. Hierarchical linear regressions that included either Session 1 cortisol (AUCg) or Session 2 cortisol (AUCg) as a moderators were conducted for males and females separately. Dummy codes were created for participant group ( $M C I=1, N A=0)$, cortisol data were $\log _{10}$ transformed and standardized, and all other data were standardized. Regression models with significant interactions are reported.

\section{Results}

\section{Males with aMCI had higher levels of cortisol than NA males during the test sessions.}

Cortisol levels during the two test sessions were analyzed separately as the psychosocial stressor (TSST) was conducted during Session 2. For Session 1 (without the TSST), males with aMCI had significantly higher levels of cortisol than any other group at the first time point (10:10AM) (all $p s<0.001$; Cohens d: for NA males=0.79, NA females=1.99, and aMCI females=1.67); time by group by sex interaction: $\mathrm{F}_{(1,27)}=3.65, p=0.066, \eta_{\mathrm{p}}=0.12$; Figure $2 \mathrm{~A}$ ). There were also main effects of sex and time (main effect of sex: $\mathrm{F}_{(1,27)}=16.24, p<0.001, \eta_{\mathrm{p}}=0.37$; main effect of time: $\mathrm{F}((1,27)=19.515$, $\left.p<0.0001, \eta_{\mathrm{p}}=0.42\right)$. For AUCg in Session 1, aMCI males had higher levels of cortisol than all other groups (all $p s<0.05$; Cohens d: for NA males $=0.81$, NA females $=1.57$, and aMCI females $=1.95$; interaction effect of sex by group: $\mathrm{F}_{(1,27)}=4.013, p=0.055$, $\eta_{\mathrm{p}}=0.129$; Figure $2 \mathrm{~B}$ ). There was also a main effect of $\operatorname{sex}\left(\mathrm{F}_{(1,27)}=10.25, p=0.003, \eta_{\mathrm{p}}=0.28\right)$ but not group ( $p=0.37$; see Figure $\left.2 \mathrm{~B}\right)$. There were no significant differences using AUCi ( $p>0.15$, group: $\eta_{p}=0.03$, sex $\eta_{p}=0.06$, group by sex $\eta_{p}=0.07$ ).

During Session 2, in which the TSST was performed, aMCI participants had higher cortisol levels than NA (main effect of group: $\mathrm{F}_{(1,27)}=7.86, p<0.009, \eta_{\mathrm{p}}=0.23$ ). Furthermore, males had higher 
levels of cortisol than females (main effect of sex: $\mathrm{F}_{(1,27)}=13.58, p=0.001, \eta_{\mathrm{p}}=0.33$ ). Furthermore, cortisol levels were highest 30min after the TSST was initiated compared to all other time points as expected (all $p \mathrm{~s}<0.03$; main effect of time: $\mathrm{F}_{(3,81)}=3.95, p=0.01, \eta_{\mathrm{p}}=0.13$ ). A priori analyses indicated that males with aMCI had significantly higher levels of cortisol during the first two time points in the second session prior to the TSST than all other groups (all ps $<0.001$; Cohens d: timepoint 1: for NA males=1.45, NA females=2.33, and aMCI females=2.00, timepoint 2: for NA males=1.25, NA females=2.44, and aMCI females=1.82). Importantly, males with aMCI did not show any changes in cortisol levels across Session 2 (all ps >0.18) whereas females with aMCI had significantly higher levels of cortisol 30min post-TSST than NA females ( $p=0.003$ Cohens $d=1.03$ ) but not at any other time point (all ps>0.18; Cohens $\mathrm{d}=0.4-0.98$; Figure 2C).

For AUCg, in Session 2 we found that males had higher levels of cortisol than females (main effect of sex: $\mathrm{F}_{(1,27)}=8.36, p<0.007, \eta_{\mathrm{p}}=0.24$ ) and aMCI had higher levels than NA (main effect of group: $\left.\mathrm{F}_{(1,27)}=4.202, p=0.05, \eta_{\mathrm{p}}=0.135\right)$. A priori we also found that aMCI males had higher AUCg than NA ( $p=0.060$, one-tailed, 0.03; Cohens d: for NA males=0.77, NA females=2.39, and aMCI females=1.40) which was not evident in the females ( $p=0.35$; Cohens $\mathrm{d}=0.73$ between female groups) see Figure 2D). There were no significant differences using AUCi ( $p>0.27$, group: $\eta_{\mathrm{p}}=0.00$, sex $\eta_{\mathrm{p}}=0.004$, group by sex $\left.\eta_{\mathrm{p}}=0.044\right)$.

\section{[INSERT FIGURE 2 HERE]}

\section{TSST was endorsed as anxiety provoking by females more than males with aMCI}

Both sexes in the NA group endorsed the TSST as anxiety provoking, with 57\% of participants indicating that the TSST was anxiety provoking. However, among aMCI participants $80 \%$ of females but only $11 \%$ of males indicated the TSST speech was anxiety provoking $\left(\chi^{2}=6.644, p<0.01\right.$, Table 2$)$. Of those participants who rated the TSST as anxiety provoking there was no significant difference in the rating of the anxiety level ( $p s>0.43$, main effect of group $\eta_{p}=0.001$, main effect of $\operatorname{sex}=\eta_{p}=0.002$, interaction $\eta_{\mathrm{p}}=0.07$; ratings (mean and standard deviation) and sample size of those that found the TSST anxiety provoking: NA females $(n=4)=68.25 \pm 25.8$, aMCI females $(n=4)=50.25 \pm 26.10$, NA males $(n=4)=49.75 \pm 35.9$; MCI males $(n=1)=64)$. 
Immediate recall of HVLT-R was enhanced after the TSST in Session 2 compared to Session 1 in the NA $(p=0.004)$, but no such enhancement was seen in participants with aMCI $(p=0.245$; interaction: group by session: $\mathrm{F}_{(1,27)}=7.6, p=0.010, \eta_{\mathrm{p}}=0.22$, main effect of group: $\mathrm{F}_{(1,27)}=41.4, p<0.001$, $\left.\eta_{\mathrm{p}}=0.61\right)$. Breaking this down by sex, the NA groups, regardless of sex, showed enhanced recall in Session 2 compared to Session 1 (males ( $p=0.02$, Cohens $\mathrm{d}=1.14$ ) females $(p=0.04$, Cohens $\mathrm{d}=0.61)$ ). However, aMCI males had impaired immediate recall ( $p=0.04$, Cohen's $\mathrm{d}=0.63)$ on Session 2 following the stressor compared to Session 1, with no significant enhancement in the females with aMCI across sessions ( $p=0.47$; Cohen’s $\mathrm{d}=0.25$, Figure $3 \mathrm{~A}$ ). This enhancement in recall following the TSST was also seen for delayed recall for the HVLT-R which was administered 40 minutes after the TSST with all groups scoring better on Session 2 than Session 1 (main effect of session $F_{(1,27)}=7.15$, $\left.p=0.012, \eta_{\mathrm{p}}=0.21\right)$ and with aMCI scoring worse than $\mathrm{NA}\left(\mathrm{F}_{(1,27)}=63.22, p<0.001, \eta_{\mathrm{p}}=0.70\right.$; Figure 3B).

For HVLT-R retention, aMCI participants performed worse compared to NA participants regardless of 384 session (main effect of group $\mathrm{F}_{(1,26)}=27.09, p<0.001$, $\eta_{\mathrm{p}}=0.51$; Figure 3C).

\section{[INSERT FIGURE 3 HERE]}

For associative memory as expected aMCI participants remembered fewer face-name pairs than NA (main effect of group: $F_{(1,27)}=32.86, p<0.000, \eta_{p}=0.55$ ). There were no other main or interaction effects (all ps>0.25).

During the spatial working memory across sessions and trials, aMCI participants made more choices than NA across all trials (main effect of group: $\mathrm{F}_{(1,27)}=13.6, p=0.001, \eta_{\mathrm{p}}=0.34$ ). Furthermore, all participants required fewer choices by the delay trial (all $p s<0.02$; main effect of trial $\left(\mathrm{F}_{(2,52)}=4.80\right.$, $p=0.012, \eta_{\mathrm{p}}=0.16$ ). There were no other significant main effects or interactions (all $p s>0.20$; all $\eta_{\mathrm{p}}$

\section{[INSERT FIGURE 4 HERE]}

\section{Diurnal CORT did not differ between male groups}

Only 21 of 31 participants completed all five time points for cortisol collection across three consecutive days. We used multiple imputation to calculate missing values for all individuals with more than $75 \%$ of the data available (2 participants only had 2/15 and one had 9/15 samples available). Curiously these 3 participants were females with aMCI. As we did not want to use the imputed values for so many missing datapoints we performed the analyses only with males. Analysis of these 16 participants revealed only a main effect of time $\left(\mathrm{F}_{(4,56)}=48.51, p<0.001, \eta_{\mathrm{p}}=0.776\right.$; Figure $\left.5 \mathrm{~A}\right)$, but no 
403

404

405

406

407

408

409

410

411

412

413

414

415

416

417

418

419

420

421

422

423

424

425

426

427

428

429

430

431

432

433

434

main effect of group $\left(\mathrm{F}_{(1,14)}=0.0349, p=0.85, \eta_{\mathrm{p}}=0.002\right.$ or interaction $\left(\mathrm{F}_{(4,56)}=0.49, p=0.74, \eta_{\mathrm{p}}=0.033\right)$.

Post hoc analyses revealed that cortisol was higher at each early timepoint than all other timepoints except there was no difference between the two evening samples. There were no significant differences between groups in time of awakening.

We also calculated average cortisol AUCg and AUCi across all three days in males only. There were no significant effects for AUCg $\left(p=0.549, \eta_{p}=0.03\right)$ or $\operatorname{AUCi}\left(p=0.6, \eta_{p}=0.02\right)$.

\section{[INSERT FIGURE 5 HERE]}

\section{Session 1 Cortisol (AUCg) was associated with better spatial working memory in aMCI females, but} the reverse in $\mathrm{NA}$ females

We next correlated Session 1 and Session 2 AUCg with episodic, associative and spatial working memory measures across group and sex, as we saw no significant differences in AUCi. Session 1 had the only significant correlations, with females showing positive associations with AUCg and better performance in spatial working memory in aMCI females (in trial 2 ( $\mathrm{r}=-0.839, p=0.037)$ and the delay trial $(\mathrm{r}=-0.952, p=0.003))$ but the opposite patterns in NA females (trial $2(\mathrm{r}=0.796, p=0.01)$ ). These correlations were significantly different $(\mathrm{z}=3.261, p=0.001)$. In NA females, AUCg was positively associated with better HVLT-retention ( $\mathrm{r}=0.7017, p=0.035)$, but negatively associated with associative memory $(\mathrm{r}=-0.703, p=0.035)$. In males, the only correlation was positive in Session 1 was associative memory and AUCg cortisol in aMCI males ( $\mathrm{r}=0.818, p=0.007)$. There were no other significant correlations in males or for data in Session 2.

\section{Session 1 cortisol (AUCg) moderates the relationship between aMCI and episodic memory retention} in females, working memory in both females and males, and associative recognition in males.

We tested the hypothesis that the magnitude of cortisol (AUCg) moderates the relationship between participant group (aMCI, NA) and episodic (HVLT), associative (face-name), or working memory (spatial working memory) during Session 1 in males and females. Regressions revealed that higher cortisol predicted lower HVLT-R retention scores in aMCI females and higher HVLT-R retention scores in NA females (model: $F_{(3,11)}=11.64, p=0.001, R^{2}=0.760$, adjusted $R^{2}=0.695$; interaction: $\left.b=-1.944, \beta=-1.124, \mathrm{t}_{(14)}=-5.653, p<0.001, s r^{2}=0.696\right)$. Further, high Session 1 cortisol predicted a lower number of choices in aMCI females and a higher number of choices in NA females during trial 1 (model: $F_{(3,11)}=10.2, p=0.002, R^{2}=0.735$, adjusted $R^{2}=0.663$; interaction: $b=-1.527, \beta=-$ 
1.562, $\mathrm{t}_{(14)}=-4.413, p=0.001, s r^{2}=0.468$ ) and the delay trial (model: $F_{(3,11)}=11.21, p=0.001, R^{2}=0.754$, adjusted $R^{2}=0.686$; interaction: $b=-1.354, \beta=-1.228, \mathrm{t}_{(14)}=-3.593, p=0.004, s r^{2}=0.289$ ) of the spatial working memory task. In males, high Session 1 cortisol predicted increased associative recognition in aMCI males versus a decline in NA males (model: $F_{(3,12)}=9.723, p=0.002, R^{2}=0.709$, adjusted $R^{2}=0.636$; interaction: $\left.b=0.915, \beta=0.658, \mathrm{t}_{(15)}=2.466, p=0.030, s r^{2}=0.148\right)$. High Session 1 cortisol also marginally predicted a lower number of choices in aMCI males versus NA males for spatial working memory during trial 2 (model: $F_{(3,12)}=2.320, p=0.127, R^{2}=0.367$, adjusted $R^{2}=0.209$; interaction: $b=$ 1.389, $\beta=-0.856, \mathrm{t}_{(15)}=-2.177, p=0.050, s r^{2}=0.250$ ) (see Figure 6). Additionally, although no interaction was significant, group was a significant predictor for session 1 HVLT-R immediate recall and delayed recall in females and males and a predictor for face-name associative recognition in females (all

[INSERT FIGURE 6 HERE]

\section{Session 2 cortisol (AUCg) moderates the relationship between aMCI and perceptions of anxiety in} response to the TSST speech in males

We tested the hypothesis that the magnitude of Session 2 cortisol (AUCg) moderates the relationship between participant group (aMCI, NA) and episodic (HVLT), associative (face-name), working memory (spatial working memory), or perceptions of anxiety to the TSST speech during Session 2 in males and females. Regressions revealed that high Session 2 cortisol predicted low perceived TSST speech anxiety ratings in aMCI males versus high perceived TSST speech anxiety ratings in NA males (model: $F_{(3,12)}=7.106, p=0.005, R^{2}=0.640$, adjusted $R^{2}=0.550$; interaction: $b=-$ 1.118, $\beta=-0.832, \mathrm{t}_{(15)}=-2.876, p=0.014, s r^{2}=0.248$; Figure 7). Similarly in females, the interaction of lower perceived TSST speech anxiety ratings in aMCI females versus NA females with high Session 2 cortisol was close to significant (model: $F_{(3,8)}=8.287, p=0.008, R^{2}=0.757$, adjusted $R^{2}=0.665$; interaction: $b=-1.266, \beta=-0.632, \mathrm{t}_{(11)}=-2.217, p=0.057, s r^{2}=0.150$ ) (see Figure 7). The interaction also approached significance for HVLT-R delayed recall in males such that high Session 2 cortisol predicted increased Session 2 HVLT-R delayed recall in aMCI males versus no change in NA adult males (model: $F_{(3,12)}=22.995, p<0.001, R^{2}=0.852$, adjusted $R^{2}=0.815$; interaction: $b=0.597, \beta=$ $\left.0.368, \mathrm{t}_{(15)}=1.984, p=0.071, s r^{2}=0.049\right)$. Additionally, although no interaction was significant, group 
males and a predictor for Session 2 HVLT-R retention and face-name associative recognition in males (all ps < 0.05) (see Table 4).

\section{[INSERT FIGURE 7 HERE]}

\section{Discussion}

Cortisol levels were significantly higher in males with aMCI, an effect that was seen during the test sessions but not in diurnal cortisol, suggesting an effect of the test environment to elicit different cortisol responses among aMCI individuals (consistent with data in NA groups from Sindi et al., 2014). Psychosocial stress, as applied by the TSST, improved immediate verbal recall in NA, but not in participants with aMCI, in fact impairing recall in males with aMCI. Furthermore, our data revealed positive correlations between cortisol levels during Session 1 and spatial working memory in females only with opposing directions based on whether the females had aMCI or NA. Higher cortisol was related to better spatial working memory in aMCI females, but worse spatial working memory in NA females during Session 1. Exploratory moderation models revealed that cortisol moderated perceived anxiety during Session 2 and each of memory domains, dependent on sex and session. Cortisol during Session 1 moderated effects on spatial working memory in both sexes and associative recognition in males, with higher cortisol reducing performance in NA but improving performance in aMCI individuals. Cortisol during Session 1 also moderated effects of episodic memory retention in females such that high cortisol enhanced performance in aMCI but impaired performance in NA females. Stress-induced cortisol in Session 2 was associated with decreased perceived anxiety to the speech in males with aMCI but increased perceived anxiety in NA males, whereas in females cortisol moderated the effect on perceived anxiety positively in both groups but a lower anxiety rating with high cortisol in aMCI females than in NA females. While our sample size was small, our results are suggestive of sex differences in the relationship between cortisol in a testing environment and memory and perceived anxiety that depended on whether the participants were NA or had aMCI. These findings, while exploratory, suggest that sex must be considered when exploring relationships between stress biomarkers and memory.

\section{Cortisol levels are higher in males with aMCI}

Amnestic MCI males had higher salivary cortisol levels as shown in the first samples of both morning sessions conducted in the laboratory. This finding is partially consistent with past studies that 
498 found increased morning serum cortisol levels in men but not women with AD (Rasmuson et al., 2011) 499 or in salivary cortisol in individuals with MCI (Venero et al, 2013). In another study, salivary cortisol 500 levels upon awakening were significantly higher in the non-amnestic and multidomain type but not in 501 the aMCI compared to NA (Venero et al., 2013). Even though we found higher morning cortisol in 502 aMCI males in the laboratory setting, cortisol levels did not differ between aMCI males and NA from 503 diurnal samples taken at home, consistent with the Venero et al. (2013) study in which participants also 504 took home samples. Rasmuson and colleagues (2011) found increased morning cortisol in males with 505 AD compared to neurotypical participants they had a low number of participants. Nevertheless, it is 506 compelling that in our findings increased morning levels of salivary cortisol are associated with aMCI 507 in males, at least in the laboratory setting. In support of this, higher cortisol and greater variations in 508 cortisol (and correlations with hippocampal volume) are seen in NA older adults when they were tested 509 in an unfamiliar versus familiar environment (Sindi et al., 2014), suggesting that stressful environments 510 influence correlates of memory. Combined with our findings concerning the influence of stress-induced 511 cortisol on anxiety ratings in males and females with aMCI, our results suggest that further

512 investigation into sex differences in cortisol levels is necessary in individuals with aMCI, and perhaps 513 in the laboratory versus home setting.

514 What might the possible mechanisms be for the cortisol differences and moderation effects 515 between sexes, and greater impairments in males with TSST on memory? Cortisol is only one output of 516 the HPA axis and other outputs may be important to monitor such as alterations to alpha amylase. In 517 addition, here we have captured acute and diurnal cortisol and it would be important to also examine 518 the influence of allostatic load on these results as well as measures of chronic stress (Yan et al., 2018). 519 In addition, HPA is a regulator of immune and metabolic function, which has been implicated as a 520 driver of or in reaction to AD. Thus, other biomarkers such as cytokines (particularly IL-6) and C521 reactive protein (CRP) may be fruitful areas of future testing. Indeed, plasma CRP was decreased in $522 \mathrm{AD}$ and MCI of both sexes and CSF IL-16 and Il-8 differed by sex dependent on APOEe4 genotype 523 (Duarte-Guterman, Inkster, Albert, Barha, Robinson, Galea, 2020). There is a paucity of studies on 524 aging, immune, and other biomarkers that have been implicated in AD with sex as a factor that has 525 elicited calls for action (Mielke et al., 2018) and are findings provide further fodder for these calls. 
As expected, the TSST induced an increase in cortisol levels, which was associated with

530 enhanced episodic memory (verbal recall) in the HVLT-R in NA but not in aMCI participants. These

531 findings are consistent with a study by Wolf et al. (2002), which found that there were negative

532 correlations between average cortisol and immediate recall of paragraphs in MCI participants but not in

533 NA. Similarly, neurotypical older adults have been found to exhibit a positive correlation between high

534 cortisol and memory performance, whereas aMCI subjects exhibit a negative correlation (de Souza-

535 Talarico et al., 2010). Collectively, the present data and previous findings suggest that cortisol has

536 opposing relationships with memory and recall in MCI versus normal aging that may differ in

537 magnitude and direction by sex. Intriguingly we also found that aMCI males did not mount a stress-

538 induced increase in cortisol with the TSST unlike the stress-induced increase in cortisol in the females

539 with aMCI. These results are intriguing given that de Souza-Talarico et al. (2020) found that greater

540 cortisol reactivity in the TSST was related to cognitive decline characteristic of future MCI after five

541 years and their population was $80 \%$ female. This may explain why females with aMCI transition to AD

542 at a greater rate than males with aMCI. The ability of the HPA axis to mount an appropriate stress

543 response may be an important biomarker for AD transition with differences depending on sex.

\section{Sex may influence the effects stress-induced cortisol on memory in aMCI}

In a few of our findings, there were opposing effects of cortisol associations or effects of stress

between aMCI and NA dependent on sex. This is intriguing and suggests that sex should be considered

548 in future studies and research on age-related cognitive impairment. This is of particular relevance

549 considering that a number of studies investigating memory and cortisol have had an imbalance of males

550 or females in their test groups (e.g. de Souza-Talarico et al., 2010; de Souza-Talarico et al., 2020; Wolf

551 et al., 2002). Furthermore, inconsistencies in the literature around the effects of acute social stress on

552 memory likely depend on multiple factors including sex and age (for review see Hidalgo et al., 2019;

553 Yan et al., 2018). However, due to a limited sample size in the current work, our sex-based analyses are

554 exploratory and due caution should be paid when generalizing the results.

Our findings of sex differences are congruent with previous studies demonstrating

556 epidemiological, symptomatic, and physiological differences between males and females with MCI.

557 The prevalence of MCI has been found to be greater in males than females, with aMCI as the most 558 common type (Petersen et al., 2010). Furthermore, the incidence of MCI is greater in males than in

559 females (Roberts et al., 2012) and recent studies have uncovered sex-specific risk factors for MCI to 560 AD progression (Kim et al., 2015). Although MCI is more prevalent in males, females exhibit faster 
561 deterioration in cognitive and functional measures over time (Lin et al., 2015). Sex differences are also 562 evident in cognitive and neurophysiological decline with $\mathrm{AD}$, as females experience accelerated 563 hippocampal atrophy and cognitive decline with AD (Ardekani et al., 2016; Irvine et al., 2012). These 564 findings, in accord with our data, emphasise the need to account for sex differences in future research 565 in memory and cognition. Intriguingly, decreases in hippocampal volume predict progression to 566 probable AD (and MCI) in women, whereas increases in white matter hyperintensities in men predict 567 progression to MCI (Burke et al., 2019). Optimistically, there is preliminary evidence that cognitive 568 training in those with aMCI is more effective in women than men (Rahe et al., 2015).

\section{The relationship between cortisol and memory may depend on brain health}

Higher cortisol levels should not always be thought of as detrimental to brain function, particularly in the face of acute stress (McEwen, 2019). Indeed, in the present study we found enhanced delayed word-list retention on the HVLT-R in the female NA participants with high cortisol levels in Session 1 under the curve. However, the opposite relationship was found in females with aMCI, as higher cortisol under the curve was associated with reduced retention in the exploratory moderating analyses. These findings are consistent with at least two other studies (de Souza-Talarico et al., 2010; Wolf et al., 2002). De Souza-Talarico et al. (2010) showed a positive relationship between cortisol and delayed recall in NA but a negative relationship in people with MCI. Furthermore, Wolf et al. (2002) showed a negative correlation between average cortisol and immediate story recall in MCI participants but no relationship to average cortisol in NA. It is important to note that opposite patterns were seen in spatial working memory and associative memory, with high Session 1 cortisol associated with improved spatial working memory in aMCI males and females and improved associative recognition in aMCI males but impairments in both associative and spatial working memory in NA participants. During Session 2, high cortisol was associated with enhanded episodic memory (HVLT-R delayed recall) in aMCI males versus NA males, but this interaction had a low effect size. These findings in aMCI participants are in contrast to past findings of impaired cognitive performance in aMCI participants with high cortisol (Popp et al., 2015). However, it should be noted that those studies are based on high diurnal cortisol in aMCI participants and saliva samples were not collected on the same days that cognitive tasks were performed (Popp et al., 2015). Cortisol measures used in our moderation models were based on saliva samples taken in the laboratory throughout the days of Session 1 or 
brain regions recruited during the task as spatial working memory heavily recruits the prefrontal cortex,

594 episodic memory the medial temporal prefrontal cortex and hippocampus, and associative memory the 595 entorhinal cortex (Courtney et al., 1998; Eichenbaum et al., 2017). Thus, our findings suggest that HPA 596 function may be having opposing effects on memory performance in aMCI groups compared to normal 597 cognitive aging groups.

Acute psychosocial stress improved immediate episodic recall in NA but not in aMCI participants. Although cortisol was not a moderating factor on episodic memory in Session 2, this may 600 be due to a number of factors. Stress activates the HPA axis and it is possible had we measured more timepoints of salivary cortisol we may have seen a moderating effect. In addition, it is important to consider that other biomarkers of HPA activation such as corticotropin releasing hormone (CRH), adrenocorticotrophic hormone (ACTH), or sympathetic activation via the sympathetic-adrenalmedullary system (SAM: alpha amylase, epinephrine, heart rate variability) may have a moderating effect on memory with acute stress. Certainly, it is intriguing that the TSST had an adverse effect on episodic memory in aMCI but a positive effect in NA participants. Indeed, other research has found enhancing effects of the TSST on memory, depending on when TSST was administered relative to memory testing (encoding, retention, recall) that depends on age and sex (Hidalgo et al., 2019). Enhancing effects on episodic memory are see in older (middle-aged) NA women with TSST (Almela et al., 2011). Others have found no effect of cortisol during TSST to moderate working memory in older NA individuals (Pulopulos et al., 2015), consistent in part to our findings that TSST did not influence spatial working memory in the present study. Furthermore, there are well known sex

613 differences in the effects of stress in animal models (Goel et al., 2014). However, the effects of age and 614 stress on learning are not as well studied. In light of these findings, we encourage the research 615 community to make it a priority to examine sex as a factor in analyses of aging and cognition.

\section{Stress-induced cortisol in Session 2 was associated with greater ratings of anxiety in response to the}

\section{TSST speech in all groups except aMCI males which were associated with reduced anxiety}

Our models revealed that Session 2 cortisol moderated the effect of aMCI on perception of anxiety for the TSST speech in both male and female participants. Greater Session 2 increases in cortisol, the session that involved stress exposure, was associated with greater perceptions of anxiety in NA males and females and to a lesser extent in aMCI females. This finding is similar to previous studies that have found higher anxiety scores in healthy male and female participants exposed to 
625 levels of Session 2 cortisol were associated with reduced perceived anxiety in our study. Anxiety is 626 found in a high percentage of patients with MCI, subjective cognitive decline, and AD (Banning et al., 627 2020), but a previous study by Guerdoux-Ninot and Trouillet (2019) found lower perceived stress in 628 response to a Stroop test in male and female AD and aMCI participants compared to NA as the task 629 became more effortful. In the present study, a large percentage of aMCI males did not characterize the 630 TSST speech as anxiety provoking. A blunted perceived anxiety response to stress in aMCI males compared to NA males in the present study could be related to previous findings of increased apathy in male and female AD and aMCI participants compared to nonamnestic-MCI (naMCI) and NA participants (Ellison et al., 2008; Lanctôt et al., 2017). Indeed, past data has indicated that AD and MCI patients differ in the prevalence of symptoms of apathy with a greater prevalence in AD versus MCI groups (Siafarikas et al., 2018). However, these past studies did not analyse their males and females separately and could have missed effects of increased apathy driven by aMCI males. Moreover, these past studies did not examine the effects of cortisol levels on perceived stress or anxiety in aMCI groups. Nevertheless, our findings along with past findings suggest that, whereas increasing stressinduced cortisol is associated with increasing perceptions of anxiety in NA, male aMCI participants are

\section{Limitations}

This is an exploratory study given our low sample size and needs to be replicated with a larger population to examine sex-specific effects. Indeed, diurnal cortisol findings in aMCI females were missed because of a lack of samples. Missing diurnal data in aMCI females may reflect sex differences in partner support, whereby partners of females with aMCI may be less predisposed to assisting with remembering to engage in saliva sample collection than partners of males with aMCI might have been. In future, it may be important to measure perceived primary support and partner attitudes to ascertain why samples are missed. In the present study, we had only 2 aMCI females that completed all (or more than $75 \%$ ) of the diurnal saliva sampling. However, when comparing these two females to the rest of the aMCI group with incomplete diurnal samples, they did not differ in age, education, TSST anxiety ratings, or on episodic or associative memory tasks. In addition to low sample size, and as previously mentioned, taking saliva samples at different timepoints and analysing samples for HPA activation biomarkers other than cortisol (ACTH, CRH, alpha amylase, epinephrine, heart rate variability) may have resulted in different moderating effects of aMCI on memory tasks. Nevertheless, even though 
657 examined the effects of stress on memory function in aMCI versus NA participants. It should be noted

658 that while linear trend lines had the best fit for our data we also attempted polynomial regressions

659 (quadratic, cubic) for associations between cortisol and memory task performances specific to each

660 session in aMCI versus NA and in male versus female participants. In addition to linear associations,

661 some quadratic and cubic associations were also observed but the majority appeared to be driven by

662 outliers. In a future study, a larger sample size in each group would help elucidate whether the

663 association between cortisol and certain memory performance may be non-linear depending on aMCI

664 and/or sex. All in all, a larger sample size would help account for some of the losses in home cortisol

665 sampling and improve the power of our statistics to examine the influence of multiple other factors on 666 our data.

667 A larger sample size would also permit examination of possible aMCI phenotypes. Other

668 researchers have demonstrated heterogeneity within the aMCI subtype on memory performance

669 measures that may be predictive of progression to AD type dementia (e.g., Sanborn et al., 2017). We

670 chose to focus on the aMCI subtype in order to limit the possible influence of heterogeneous

671 underlying neuropathologies to that of incipient AD. Widening the lens to include other MCI subtypes

672 that are more likely to be of mixed etiology may produce different relationships between cortisol and

673 memory than those demonstrated here. For example, as previously described, Guerdoux-Ninot and

674 Trouillet (2019) found that effort increased perceived stress in NA adults and non-amnestic MCI

675 patients but reduced it in aMCI and AD. Thus, it would be interesting to examine larger sample sizes

676 that include MCI subtypes in a future study in order to further appreciate differences between subtypes

677 and to further explore research demonstrating MCI subtypes may have different phenotypes (Edmonds

678 et al., 2019; Sanborn et al., 2017). Certainly, our data demonstrate it would be important to consider the

679 heterogeneity of aMCI and to determine whether biological sex may be a contributing factor to the

680 heterogeneity.

681

682 Conclusions

683 The present study found relationships with cortisol (stress-induced and morning session 684 cortisol) and aMCI and moderating effects of cortisol on some domains of memory. As expected, the 685 aMCI participants performed more poorly across the memory measures as compared to NA; however 686 within this we found response patterns influenced by biological sex. We found that males with aMCI 687 had higher cortisol levels in the morning during the test sessions. We also found stress-induced 688 impairment with episodic memory only in males with aMCI. Although our sex-based analyses are 
689

690

691

692

693

694

695

696

697

698

699

700

701

702

703

704

705

706

707

708

709

710

711

712

713

exploratory due to the low sample size, sex differences were nonetheless observed. It is critical that future studies explore sex as a biological variable as we have presented evidence herein suggesting that effects at the confluence of aMCI and stress can be obfuscated or otherwise eliminated when males and females are combined instead of being considered separately. For real understanding and advancement to take place in this field, biological sex must be considered and statistically analyzed.

Estimates of the prevalence of MCI in the elderly show high variability, ranging from 3-42\% (Ward et al., 2012), due to differences in study methodology, especially with regards to the sample population (age, ethnicity, education-level, etc.) (Ward et al., 2012). Regardless, there is a health care burden associated with MCI (Ton et al., 2017) as those with MCI are more likely to develop AD (Busse et al., 2003; Lupien et al., 1998) and the health care burden of AD is more severe than that of MCI (Ton et al., 2017). The findings presented here indicate future studies should make examining sex differences (their nature, underlying mechanisms, outcomes, etc.) in aMCI a priority, as well as expand upon the influence of cortisol in aMCI and the interactions between these factors.

\section{Acknowledgments}

We gratefully acknowledge the participants for generously volunteering their time and effort. We thank Triti Namiranian, Angelina Polsinelli, Nicole D’Souza, Diana Smith, Preeyam Parikha, and Tallinn Splinter for assistance with data collection and management and Elizabeth Perez for editorial assistance.

\section{Funding and Disclosure of Interest}

This study was supported by grants from the Canadian Institutes of Health Research (CIHR-IA \#131486 to LAMG, KJM, and AKT) and by the Morris Goldenberg Medical Research Endowment (to $\mathrm{KJM}$ ). The funders had no role in the study design, collection, analyses or interpretation of the data. The authors have no further conflicts to disclose. 


\section{References}

715 Adam, E. K., Quinn, M. E., Tavernier, R., McQuillan, M. T., Dahlke, K. A., \& Gilbert, K. E. (2017). Diurnal cortisol slopes and mental and physical health outcomes: A systematic review and meta-analysis. Psychoneuroendocrinology, 83, 25-41. doi:10.1016/j.psyneuen.2017.05.018

Aggarwal, N. T., Wilson, R. S., Beck, T. L., Rajan, K. B., Mendes de Leon, C. F., Evans, D. A., \& 725 Everson-Rose, S. A. (2014). Perceived stress and change in cognitive function among adults 65 years and older. Psychosomatic Medicine, 76(1), 80-85. doi: 10.1097/PSY.0000000000000016 Holtzman, D. M., Jagust, W. J., Petersen, R. C., Snyder, P. J., Carrillo, M. C., Thies, B., \& Phelps, C. H. (2011). The diagnosis of mild cognitive impairment due to Alzheimer's disease:

Ardekani, B. A., Convit, A., \& Bachman, A. H. (2016). Analysis of the MIRIAD data shows Sex differences in hippocampal atrophy progression. Journal of Alzheimer's Disease: JAD, 50(3), 847-857. https://doi.org/10.3233/JAD-150780

Burke, S. L., Hu, T., Fava, N. M., Li, T., Rodriguez, M. J., Schuldiner, K. L., Burgess, A., \& Laird, A. (2019). Sex differences in the development of mild cognitive impairment and probable Alzheimer's disease as predicted by hippocampal volume or white matter hyperintensities. Journal of Women \& Aging, 31(2), 140-164. https://doi.org/10.1080/08952841.2018.1419476 
746

747

748

749

750

751

752

753

754

755

756

757

758

759

760

761

762

763

764

765

766

767

768

769

770

771

772

773

774

775

776

Busse, A., Bischkopf, J., Riedel-Heller, S. G., \& Angermeyer, M. C. (2003). Mild cognitive impairment: prevalence and incidence according to different diagnostic criteria. Results of the Leipzig Longitudinal Study of the Aged (LEILA75+). The British Journal of Psychiatry: The Journal of Mental Science, 182, 449-454.

Büttler, R. M., Bagci, E., Brand, H. S., Heijer, M. den, Blankenstein, M. A., \& Heijboer, A. C. (2018). Testosterone, androstenedione, cortisol and cortisone levels in human unstimulated, stimulated and parotid saliva. Steroids, 138, 26-34. https://doi.org/10.1016/j.steroids.2018.05.013

Courtney, S. M., Petit, L., Maisog, J. M., Ungerleider, L. G., \& Haxby, J. V. (1998). An area specialized for spatial working memory in human frontal cortex. Science, 279(5355), 13471351. https://doi.org/10.1126/science.279.5355.1347

Cullum, S., Huppert, F. A., McGee, M., Dening, T., Ahmed, A., Paykel, E. S., \& Brayne, C. (2000). Decline across different domains of cognitive function in normal ageing: Results of a longitudinal population-based study using CAMCOG. International Journal of Geriatric Psychiatry, 15(9), 853-862.

Dedovic, K., Duchesne, A., Andrews, J., Engert, V., \& Pruessner, J. C. (2009). The brain and the stress axis: The neural correlates of cortisol regulation in response to stress. NeuroImage, 47(3), 864871. https://doi.org/10.1016/j.neuroimage.2009.05.074

Delis D, Kaplan E, \& Kramer JH. Delis-Kaplan Executive Function System ${ }^{T M}$. San Antonio: Psychological Corporation; 2001.

de Souza-Talarico, J. N., Alves, A. R., Brucki, S., Nitrini, R., Lupien, S. J., \& Suchecki, D. (2020). Cortisol reactivity to a psychosocial stressor significantly increases the risk of developing Cognitive Impairment no Dementia five years later. Psychoneuroendocrinology, 115, 104601. https://doi.org/10.1016/j.psyneuen.2020.104601

de Souza-Talarico, J. N., Chaves, E. C., Lupien, S. J., Nitrini, R., \& Caramelli, P. (2010). Relationship between cortisol levels and memory performance may be modulated by the presence or absence of cognitive impairment: Evidence from healthy elderly, mild cognitive impairment and Alzheimer's disease subjects. Journal of Alzheimer's Disease: JAD, 19(3), 839-848. https://doi.org/10.3233/JAD-2010-1282

Dijckmans, B., Tortosa-Martínez, J., Caus, N., González-Caballero, G., Martínez-Pelegrin, B., Manchado-Lopez, C., Cortell-Tormo, J. M., Chulvi-Medrano, I., \& Clow, A. (2017). Does the diurnal cycle of cortisol explain the relationship between physical performance and cognitive 
777

778

779

780

781

782

783

784

785

786

787

788

789

790

791

792

793

794

795

796

797

798

799

800

801

802

803

804

805

806

807

808

function in older adults?. European review of aging and physical activity : official journal of the European Group for Research into Elderly and Physical Activity, 14, 6. .

Doecke, J. D., Laws, S. M., Faux, N. G., Wilson, W., Burnham S. C., Lam, C-P., Mondal, A., Bedo, J., Bush, A. I., Brown, B., De Ruyck, K., Ellis, K. A., Fowler, C., Gupta, V. B., Head, R., Macaulay, S. L., Pertile, K., Rowe, C. C., Rembach, A., ... Australian Imaging Biomarker and Lifestyle Research Group. (2012). Blood-based protein biomarkers for diagnosis of Alzheimer disease. Archives of Neurology, 69(10), 1318-1325. doi:10.1001/archneurol.2012.1282

Donaldson, W. (1992). Measuring recognition memory. Journal of Experimental Psychology: General, 121(3), 275-277. https://doi.org/10.1037/0096-3445.121.3.275

Duarte-Guterman, P., Inkster A. M., Albert A. Y., Barha, C. K., Robinson, W. P., Galea L. A. M., \& Alzheimer's Disease Neuroimaging Initiative. (2020). Inflammation and epigenetic age in Alzheimer's disease: Do sex and APOE matter? bioRxiv, 741777v4. https://doi.org/10.1101/741777

Duff, S. J., \& Hampson, E. (2001). A sex difference on a novel spatial working memory task in humans. Brain and Cognition, 47(3), 470-493. https://doi.org/10.1006/brcg.2001.1326

Edmonds, E. C., McDonald, C. R., Marshall, A., Thomas, K. R., Eppig, J., Weigand, A. J., DelanoWood, L., Galasko, D. R., Salmon, D. P., Bondi, M. W., \& Alzheimer's Disease Neuroimaging Initiative. (2019). Early versus late MCI: Improved MCI staging using a neuropsychological approach. Alzheimer's \& Dementia, 15(5), 699-708. doi:10.1016/j.jalz.2018.12.009

Eichenbaum, H. (2017). Prefrontal-hippocampal interactions in episodic memory. Nature Reviews Neuroscience, 18(9), 547-558. https://doi.org/10.1038/nrn.2017.74

Ellenbogen, M. A., Schwartzman, A. E., Stewart, J., \& Walker, C. D. (2002). Stress and selective attention: The interplay of mood, cortisol levels, and emotional information processing. Psychophysiology, 39(6), 723-732.

Ellison, J. M., Harper, D. G., Berlow, Y., \& Zeranski, L. (2008). Beyond the “C” in MCI: noncognitive symptoms in amnestic and non-amnestic mild cognitive impairment. CNS Spectrums, 13(1), 66-72. https://doi.org/10.1017/s1092852900016175

Elosúa, M. R., \& Contreras, M. J. (2017). Gender differences in verbal and visuospatial working memory tasks in patients with mild cognitive impairment and Alzheimer disease. Dementia and Geriatric Cognitive Disorders Extra, 7(1), 101-108.

Ferrari, E., Cravello, L., Muzzoni, B., Casarotti, D., Paltro, M., Solerte, S. B., Fioravanti, M., Cuzzoni, G., Pontiggia, B., \& Magri, F. (2001). Age-related changes of the hypothalamic-pituitary- 
809

810

811

812

813

814

815

816

817

818

819

820

821

822

823

824

825

826

827

828

829

830

831

832

833

834

835

836

837

838

839

840

adrenal axis: Pathophysiological correlates. European Journal of Endocrinology, 144(4), 319329. doi:10.1530/eje.0.1440319.

Gale, S. D., Baxter, L., \& Thompson, J. (2016). Greater memory impairment in dementing females than males relative to sex-matched healthy controls. Journal of Clinical and Experimental Neuropsychology, 38(5), 527-533. https://doi.org/10.1080/13803395.2015.1132298

Goel, N., Workman, J. L., Lee, T. T., Innala, L., \& Viau, V. (2014). Sex differences in the HPA axis. Comprehensive Physiology, 4(3), 1121-1155. https://doi.org/10.1002/cphy.c130054

Grady, C. (2012) Trends in Neurocognitive Aging. Nat Rev Neurosci,13(7), 491-505.

Guerdoux-Ninot, E. \& Trouillet, R. (2019). Impact of perceived stress on cognitive performance: Moderating effect of mild cognitive impairment and Alzheimer's disease. Journal of Clinical and Experimental Neuropsychology, 41(4), 364-379. doi:10.1080/13803395.2018.1564250

Hartmann, A., Veldhuis, J. D., Deuschle, M., Standhardt, H., \& Heuser, I. (1997). Twenty-four hour cortisol release profiles in patients with Alzheimer's and Parkinson's disease compared to normal controls: Ultradian secretory pulsatility and diurnal variation. Neurobiology of Aging, 18(3), 285-289.

Hidalgo, V., Pulopulos, M. M., \& Salvador, A. (2019). Acute psychosocial stress effects on memory performance: Relevance of age and sex. Neurobiology of Learning and Memory, 157, 48-60. https://doi.org/10.1016/j.nlm.2018.11.013

Irvine, K., Laws, K. R., Gale, T. M., \& Kondel, T. K. (2012). Greater cognitive deterioration in women than men with Alzheimer's disease: A meta analysis. Journal of Clinical and Experimental Neuropsychology, 34(9), 989-998. https://doi.org/10.1080/13803395.2012.712676

Jack, C. R. Jr., Therneau, T. M., Weigand, S. D., Wiste, H. J., Knopman, D. S., Vemuri, P., Lowe, V. J., Mielke, M. M., Roberts, R. O., Machulda, M. M., Graff-Radford, J., Jones, D. T., Schwarz, C. G., Gunter, J. L., Senjem, M. L., Rocca, W. A., \& Petersen, R. C. (2019). Prevalence of biologically vs clinically defined Alzheimer spectrum entities using the National Institute on Aging-Alzheimer's Association research framework. JAMA Neurology, 76(10), 1174-1183. https://doi.org/10.1001/jamaneurol.2019.1971

Justice N. J. (2018). The relationship between stress and Alzheimer's disease. Neurobiology of stress, 8, 127-133. https://doi-org.ezproxy.library.ubc.ca/10.1016/j.ynstr.2018.04.002

Kim, S., Kim, M. J., Kim, S., Kang, H. S., Lim, S. W., Myung, W., Lee, Y., Hong, C. H., Choi S. H., Na, D. L., Seo, S. W., Ku, B. D., Kim, S. Y., Kim, S. Y., Jeong, J. H., Park, S. A., Carroll, B. J., \& Kim, D. K. (2015). Gender differences in risk factors for transition from mild cognitive 
841

842

843

844

845

846

847

848

849

850

851

852

853

854

855

856

857

858

859

860

861

862

863

864

865

866

867

868

869

870

871

872

impairment to Alzheimer's disease: A CREDOS study. Comprehensive Psychiatry, 62, 114122. https://doi.org/10.1016/j.comppsych.2015.07.002

Kirschbaum, C., Pirke, K. M., \& Hellhammer, D. H. (1993). The 'Trier Social Stress Test'--A tool for investigating psychobiological stress responses in a laboratory setting. Neuropsychobiology, 28(1-2), 76-81. https://doi.org/10.1159/000119004

Koran, M. E. I., Wagener, M., Hohman, T. J., \& Alzheimer’s Neuroimaging Initiative. (2017). Sex differences in the association between AD biomarkers and cognitive decline. Brain Imaging and Behavior, 11(1), 205-213. https://doi.org/10.1007/s11682-016-9523-8

Kudielka, B. M., \& Kirschbaum, C. (2005). Sex differences in HPA axis responses to stress: A review. Biological Psychology, 69(1), 113-132. https://doi.org/10.1016/j.biopsycho.2004.11.009

Lanctôt, K. L., Agüera-Ortiz, L., Brodaty, H., Francis, P. T., Geda, Y. E., Ismail, Z., Marshall, G. A., Mortby, M. E., Onyike, C. U., Padala, P. R., Politis, A. M., Rosenberg, P. B., Siegel, E., Sultzer, D. L., \& Abraham, E. H. (2017). Apathy associated with neurocognitive disorders: recent progress and future directions. Alzheimer's \& Dementia, 13(1), 84-100.

Laske C., Leyhe T., Stransky E., Hoffmann N., Fallgatter A. J., \& Dietzsch J., (2011). Identification of a blood-based biomarker panel for classification of Alzheimer's disease. International Journal of Neuropsychopharmacology, 14(9), 1147-1155.

Laughlin, G. A., \& Barrett-Connor, E. (2000). Sexual dimorphism in the influence of advanced aging on adrenal hormone levels: The Rancho Bernardo Study. The Journal of Clinical Endocrinology and Metabolism, 85(10), 3561-3568. https://doi.org/10.1210/jcem.85.10.6861

Lin, K. A., Choudhury, K. R., Rathakrishnan, B. G., Marks, D. M., Petrella, J. R., Doraiswamy, P. M., \& Alzheimer’s Disease Neuroimaging Initiative. (2015). Marked gender differences in progression of mild cognitive impairment over 8 years. Alzheimer's \& Dementia (New York, $N$. Y.), 1(2), 103-110. https://doi.org/10.1016/j.trci.2015.07.001

Lupien, S. J., de Leon, M., de Santi, S., Convit, A., Tarshish, C., Nair, N. P., Thakur, M., McEwen, B. S., Hauger, R. L., \& Meaney, M. J. (1998). Cortisol levels during human aging predict hippocampal atrophy and memory deficits. Nature Neuroscience, 1(1), 69-73. https://doi.org/10.1038/271

Mah, L., Binns, M. A., Steffens, D. C., \& Alzheimer's Disease Neuroimaging Initiative. (2015). Anxiety symptoms in amnestic mild cognitive impairment are associated with medial temporal atrophy and predict conversion to Alzheimer disease. The American Journal of Geriatric Psychiatry, 23(5), 466-476. 
873

874

875

876

877

878

879

880

881

882

883

884

885

886

887

888

889

890

891

892

893

894

895

896

897

898

899

900

901

902

903

Mayes, A. R., Holdstock, J. S., Isaac, C. L., Montaldi, D., Grigor, J., Gummer, A., Cariga, P., Downes, J. J., Tsivilis, D., Gaffan, D., Gong, Q., \& Norman, K. A. (2004). Associative recognition in a patient with selective hippocampal lesions and relatively normal item recognition.

Hippocampus, 14(6), 763-784. https://doi.org/10.1002/hipo.10211

McEwen B. S. (2019). The good side of "stress". Stress (Amsterdam, Netherlands), 22(5), 524-525. https://doi.org/10.1080/10253890.2019.1631794

Mielke, M. M., Ferretti, M. T., Iulita, M. F., Hayden, K., \& Khachaturian, A. S. (2018). Sex and gender in Alzheimer's disease - Does it matter? Alzheimer's \& Dementia, 14(9), 1101-1103.

Mielke, M. M., Vemuri, P., \& Rocca, W. A. (2014). Clinical epidemiology of Alzheimer’s disease: Assessing sex and gender differences. Clinical Epidemiology, 6, 37-48. https://doi.org/10.2147/CLEP.S37929

Nebel, R. A., Aggarwal, N. T., Barnes, L. L., Gallagher, A., Goldstein, J. M., Kantarci, K., Mallampalli, M. P., Mormino, E. C., Scott, L., Yu, W. H., Maki, P. M., \& Mielke, M. M. (2018). Understanding the impact of sex and gender in Alzheimer's disease: A call to action. Alzheimer's \& Dementia, 14(9), 1171-1183. https://doi.org/10.1016/j.jalz.2018.04.008

Norman, R. M. G., Gawronski, B., Hampson, E., Sorrentino, R. M., Szeto, A., \& Ye, Y. (2010). Physical proximity in anticipation of meeting someone with schizophrenia: The role of explicit evaluations, implicit evaluations and cortisol levels. Schizophrenia Research, 124(1-3), 74-80. https://doi.org/10.1016/j.schres.2010.07.021

Otte, C., Hart, S., Neylan, T. C., Marmar, C. R., Yaffe, K., \& Mohr, D. C. (2005). A meta-analysis of cortisol response to challenge in human aging: importance of gender.

Psychoneuroendocrinology, 30(1), 80-91. https://doi.org/10.1016/j.psyneuen.2004.06.002

Petersen, R. C. (2004). Mild cognitive impairment as a diagnostic entity. Journal of Internal Medicine, 256, 183-194.

Petersen, R. C., Roberts, R. O., Knopman, D. S., Geda, Y. E., Cha, R. H., Pankratz, V. S., Boeve, B. F., Tangalos, E. G., Ivnik, R. J., \& Rocca, W. A. (2010). Prevalence of mild cognitive impairment is higher in men. The Mayo Clinic Study of Aging. Neurology, 75(10), 889-897. https://doi.org/10.1212/WNL.0b013e3181f11d85

Popp, J., Wolfsgruber, S., Heuser, I., Peters, O., Hüll, M., Schröder, J., Möller, H-J., Lewczuk, P., Schneider, A., Jahn, H., Luckhaus, C., Perneczky, R., Frölich, L., Wagner, M., Maier, W., Wiltfang, J., Kornhuber, J., \& Jessen, F. (2015). Cerebrospinal fluid cortisol and clinical disease 
904

905

906

907

908

909

910

911

912

913

914

915

916

917

918

919

920

921

922

923

924

925

926

927

928

929

930

931

932

933

934

progression in MCI and dementia of Alzheimer's type. Neurobiology of Aging, 36(2), 601-607. https://doi.org/10.1016/j.neurobiolaging.2014.10.031

Pruessner, J. C., Kirschbaum, C., Meinlschmid, G., \& Hellhammer, D. H. (2003). Two formulas for computation of the area under the curve represent measures of total hormone concentration versus time-dependent change. Psychoneuroendocrinology, 28(7), 916-931.

Pulopulos, M. M., Hidalgo, V., Almela, M., Puig-Perez, S., Villada, C., \& Salvador, A. (2015). Acute stress and working memory in older people. Stress, 18(2), 178-187.

Rahe, J., Liesk, J., Rosen, J. B., Petrelli, A., Kaesberg, S., Onur, O. A., Kessler, J., Fink, G. R., \& Kalbe, E. (2015). Sex differences in cognitive training effects of patients with amnestic mild cognitive impairment. Neuropsychology, Development, and Cognition. Section B, Aging, Neuropsychology and Cognition, 22(5), 620-638. https://doi.org/10.1080/13825585.2015.1028883

Rasmuson, S., Näsman, B., \& Olsson, T. (2011). Increased serum levels of dehydroepiandrosterone (DHEA) and interleukin-6 (IL-6) in women with mild to moderate Alzheimer's disease. International Psychogeriatrics, 23(9), 1386-1392. doi:10.1017/S1041610211000810

Raz, N. \& Rodrigue, K. M. (2006). Differential aging of the brain: patterns, cognitive correlates and modifiers. Neuroscience and Biobehavioral Reviews, 30(6), 730-748. https://doi.org/10.1016/j.neubiorev.2006.07.001

Roberts, R. O., Geda, Y. E., Knopman, D. S., Cha, R. H., Pankratz, V. S., Boeve, B. F., Tangalos, E. G., Ivnik, R. J., Rocca, W. A., \& Petersen, R. C. (2012). The incidence of MCI differs by subtype and is higher in men: The Mayo Clinic Study of Aging. Neurology, 78(5), 342-351. https://doi.org/10.1212/WNL.0b013e3182452862

Sanborn V, Putcha D, \& Tremont, G. (2017). Correlates of recognition memory performance in amnestic mild cognitive impairment. Journal of Clinical and Experimental Neuropsychology, 40(2), 205-211. doi:10.1080/13803395.2017.1334043

Shi, F., Liu, B., Zhou, Y., Yu, C., \& Jiang, T. (2009). Hippocampal volume and asymmetry in mild cognitive impairment and Alzheimer's disease: Meta-analyses of MRI studies. Hippocampus, 19(11), 1055-1064. https://doi.org/10.1002/hipo.20573

Siafarikas, N., Selbaek, G., Fladby, T., Benth, J. Š., Auning, E., \& Aarsland, D. (2018). Frequency and subgroups of neuropsychiatric symptoms in mild cognitive impairment and different stages of dementia in Alzheimer's disease. International Psychogeriatrics, 30(1), 103-113. 
935

936

937

938

939

940

941

942

943

944

945

946

947

948

949

950

951

952

953

954

955

956

957

958

959

960

961

962

963

964

965

966

Sindi, S., Fiocco, A. J., Juster, R. P., Lord, C., Pruessner, J., \& Lupien, S. J. (2014). Now you see it, now you don't: Testing environments modulate the association between hippocampal volume and cortisol levels in young and older adults. Hippocampus, 24(12), 1623-1632.

Spreen O, Strauss E. A Compendium of Neuropsychological Tests: Administration, Norms, and Commentary, 2nd Ed. New York, NY, US: Oxford University Press; 1998.

Sohn, D., Shpanskaya, K., Lucas, J. E., Petrella, J. R., Saykin, A. J., Tanzi, R. E., Samatova, N. F., \& Doraiswamy, P. M. (2018). Sex differences in cognitive decline in subjects with high likelihood of mild cognitive impairment due to Alzheimer’s disease. Scientific Reports, 8(1), 7490. https://doi.org/10.1038/s41598-018-25377-w

Spreen, O., \& Strauss, E. (1998). A compendium of neuropsychological tests: Administration, norms, and commentary (2nd ed.). New York: Oxford University Press.

Sundermann, E. E., Biegon, A., Rubin, L. H., Lipton, R. B., Landau, S., Maki, P. M., \& Alzheimer’s Disease Neuroimaging Initiative. (2017). Does the female advantage in verbal memory contribute to underestimating Alzheimer's Disease pathology in women versus men? Journal of Alzheimer's Disease: JAD, 56(3), 947-957. https://doi.org/10.3233/JAD-160716

Ton, T. G. N., DeLeire, T., May, S. G., Hou, N., Tebeka, M. G., Chen, E., \& Chodosh, J. (2017). The financial burden and health care utilization patterns associated with amnestic mild cognitive impairment. Alzheimer's \& Dementia, 13(3), 217-224. https://doi.org/10.1016/j.jalz.2016.08.009

Tortosa-Martínez, J., Manchado, C., Cortell-Tormo, J. M., \& Chulvi-Medrano, I. (2018). Exercise, the diurnal cycle of cortisol and cognitive impairment in older adults. Neurobiology of stress, 9, 40-47. https://doi-org.ezproxy.library.ubc.ca/10.1016/j.ynstr.2018.08.004

Troyer, A. K., D’Souza, N. A., Vandermorris, S., \& Murphy, K. J. (2011). Age-related differences in associative memory depend on the types of associations that are formed. Neuropsychology, Development, and Cognition. Section B, Aging, Neuropsychology and Cognition, 18(3), 340352. https://doi.org/10.1080/13825585.2011.553273

Troyer, A. K., Murphy, K. J., Anderson, N. D., Craik, F. I. M., Moscovitch, M., Maione, A., \& Gao, F. (2012). Associative recognition in mild cognitive impairment: Relationship to hippocampal volume and apolipoprotein E. Neuropsychologia, 50(14), 3721-3728. https://doi.org/10.1016/j.neuropsychologia.2012.10.018

Troyer, A. K., Murphy, K. J., Anderson, N. D., Hayman-Abello, B. A., Craik, F. I. M., \& Moscovitch, M. (2008). Item and associative memory in amnestic mild cognitive impairment: Performance 
on standardized memory tests. Neuropsychology, 22(1), 10-16. https://doi.org/10.1037/0894-

969 Venero, C., Díaz-Mardomingo, C., Pereda-Pérez, I., García-Herranz, S., Utrera, L., Valencia, A., \& Peraita, H. (2013). Increased morning salivary cortisol levels in older adults with nonamnestic and multidomain mild cognitive impairment. Psychoneuroendocrinology, 38(4), 488-498. https://doi.org/10.1016/j.psyneuen.2012.07.007

973 Wang, L. Y., Raskind, M. A., Wilkinson, C. W., Shofer, J. B., Sikkema, C., Szot, P., Quinn, J. F., 974 Galasko, D. R., \& Peskind, E. R. (2018). Associations between CSF cortisol and CSF norepinephrine in cognitively normal controls and patients with amnestic MCI and AD dementia. International Journal of Geriatric Psychiatry, 33(5), 763-768. https://doi.org/10.1002/gps.4856

979

980

Ward, A., Arrighi, H. M., Michels, S., \& Cedarbaum, J. M. (2012). Mild cognitive impairment: Disparity of incidence and prevalence estimates. Alzheimer's \& Dementia, 8(1), 14-21. https://doi.org/10.1016/j.jalz.2011.01.002

Wolf, O. T., Convit, A., Thorn, E., \& de Leon, M. J. (2002). Salivary cortisol day profiles in elderly with mild cognitive impairment. Psychoneuroendocrinology, 27(7), 777-789.

Yan, Y., Dominguez, S., Fisher, D. W., \& Dong, H. (2018). Sex differences in chronic stress responses and Alzheimer's disease. Neurobiology of stress, 8, 120-126. 
986 Table 1 Descriptive Data for the Participant Groups

\begin{tabular}{|c|c|c|c|}
\hline & $\begin{array}{c}\text { NA } \\
(n=15)\end{array}$ & $\begin{array}{c}\mathrm{aMCI} \\
(n=16)\end{array}$ & Cohen's $d$ \\
\hline Age (years) & $75.3(8.7)$ & $74.6(8.0)$ & 0.08 \\
\hline Female:Male ratio & $9: 7$ & $6: 9$ & \\
\hline Education (years) & $14.4(3.5)$ & $14.9(3.0)$ & 0.15 \\
\hline MMSE & $29.0(1.0)$ & $27.7(1.9)$ & 0.89 \\
\hline Vocabulary SS & $13.6(2.9)$ & $13.8(2.7)$ & 0.07 \\
\hline Digit Span Forward SS & $11.9(3.4)$ & $10.8(2.9)$ & 0.34 \\
\hline $\begin{array}{l}\text { Digits Span Backward } \\
\text { SS }\end{array}$ & $13.3(3.3)$ & $12.7(2.1)$ & 0.01 \\
\hline TMT A SS & $10.3(1.48)$ & $9.47(1.92)$ & 0.48 \\
\hline TMT B SS & $12.69(1.89)$ & $10.79(1.93)$ & 0.99 \\
\hline Rey-Osterrieth Copy SS & $8.94(2.14)$ & $8.25(1.54)$ & 0.37 \\
\hline $\begin{array}{l}\text { Rey-Osterrieth } \\
\text { Immediate Memory* SS }\end{array}$ & $11.19(3.63)$ & $7.25(2.63)$ & 1.24 \\
\hline Boston Naming* SS & $12.68(2.75)$ & $10.80(3.53)$ & 0.59 \\
\hline HADS Depression Scale & $2.6(2.3)$ & $2.6(2.3)$ & 0 \\
\hline HADS Anxiety Scale & $5.1(3.8)$ & $5.6(2.5)$ & 0.16 \\
\hline
\end{tabular}
Note. Mean scores with standard deviations in parentheses. NA= normal aging; aMCI = amnestic mild cognitive impairment; MMSE = Mini-Mental Status Exam; Vocabulary = Expressive vocabulary;

989 Digits Span = Total attention span score for digits forward and backwards; TMT = Trail Making Test A 990 (number sequencing) and B (alternating number-letter sequencing); Rey-O Copy = visual construction 991 of the Rey-Osterreith Complex Figure; Rey-O Memory = immediate recall of the complex figure; SS = 992 age-corrected scaled score. HADS = Hospital Anxiety Depression Scale with scores $<7$ considered 993 within normal limits. *indicates significantly different between NA and aMCI 
995 Table 2: Demographic Data for the Participant Groups

\begin{tabular}{ccccccc}
\hline & $\begin{array}{c}\text { NA } \\
\text { males } \\
(n=7)\end{array}$ & $\begin{array}{c}\text { aMCI } \\
\text { males } \\
(n=9)\end{array}$ & $\begin{array}{c}\text { NA } \\
\text { females } \\
(n=9)\end{array}$ & $\begin{array}{c}\text { aMCI } \\
\text { females } \\
(n=6)\end{array}$ & P values & $\begin{array}{c}\text { Effect Size: } \\
\eta^{2}\end{array}$ \\
\hline $\begin{array}{c}\text { Age } \\
\text { (years)* }\end{array}$ & $(81.3$ & $76.0(7.7)$ & 79.8 & $\begin{array}{c}71.5 \\
(8.0)\end{array}$ & $\begin{array}{c}\text { Sex ns } \\
\text { Group ns } \\
\text { Group by sex }\end{array}$ & $\begin{array}{c}\text { Gex .02 } \\
\text { Group by sex .02 }\end{array}$ \\
$\begin{array}{c}\text { Education } \\
\text { (years) }\end{array}$ & 14.7 & $15.5(2.2)$ & 14.1 & 13.5 & $\begin{array}{c}0.025 \\
\text { Sex ns }\end{array}$ & Sex .05 \\
& $(3.3)$ & & $(3.6)$ & $(2.7)$ & $\begin{array}{c}\text { Group ns } \\
\text { Group by sex } \\
n s\end{array}$ & $\begin{array}{c}\text { Group .00 } \\
\text { Group by sex .01 }\end{array}$
\end{tabular}

996 Note. Mean scores with standard deviations in parentheses. NA= normal aging; aMCI = amnestic mild 997 cognitive impairment * denotes significant difference.

998

999 
1000 Table 3. Session 1 cortisol moderation models: main effects and interactions.

\begin{tabular}{|c|c|c|c|c|c|c|c|c|}
\hline & Model & Term & $B$ & $S E$ & $\beta$ & $t$ & $p$ & $s r^{2}$ \\
\hline \multirow[t]{36}{*}{ Males } & HVLT-R & GRP & - & 0.396 & - & - & $0.002 *$ & 0.468 \\
\hline & Immediate & & 1.566 & & 0.921 & 3.960 & & \\
\hline & Recall & S1BC & - & & & & & 0.010 \\
\hline & & & 0.134 & & & & & \\
\hline & & GRP $x$ & 0.407 & & & & & 0.035 \\
\hline & & S1BC & & & & & & \\
\hline & HVLT-R & GRP & - & 0.410 & - & - & $0.017 *$ & 0.160 \\
\hline & Delayed Recall & & 1.138 & & 0.538 & 2.773 & & \\
\hline & & S1BC & - & & & & & 0.016 \\
\hline & & & 0.210 & & & & & \\
\hline & & GRP $x$ & - & & & & & 0.024 \\
\hline & & S1BC & 0.415 & & & & & \\
\hline & HVLT-R & GRP & - & & & & & 0.084 \\
\hline & Retention & & 0.870 & & & & & \\
\hline & & S1BC & - & & & & & 0.002 \\
\hline & & & 0.080 & & & & & \\
\hline & & GRP x & - & & & & & 0.057 \\
\hline & & S1BC & 0.681 & & & & & \\
\hline & Associative & GRP & - & 0.391 & - & - & $<0.001 *$ & 0.593 \\
\hline & Recognition & & 1.933 & & 1.036 & 4.940 & & \\
\hline & & S1BC & - & & & & & 0.043 \\
\hline & & & 0.307 & & & & & \\
\hline & & GRP x & 0.915 & 0.371 & 0.658 & 2.466 & $0.030 *$ & 0.148 \\
\hline & & S1BC & & & & & & \\
\hline & Spatial Working & GRP & 0.809 & & & & & 0.065 \\
\hline & Memory - Trial & S1BC & 0.219 & & & & & 0.014 \\
\hline & 1 & GRP $x$ & - & & & & & 0.001 \\
\hline & & S1BC & 0.104 & & & & & \\
\hline & Spatial & GRP & 1.484 & 0.673 & 0.682 & 2.206 & 0.048* & 0.257 \\
\hline & Working & S1BC & 0.549 & & & & & 0.102 \\
\hline & Memory - & GRP x & - & 0.638 & - & & $0.050 *$ & 0.250 \\
\hline & Trial 2 & S1BC & 1.389 & & 0.856 & 2.177 & & \\
\hline & Spatial Working & GRP & 1.337 & & & & & 0.188 \\
\hline & Memory - & S1BC & 0.177 & & & & & 0.010 \\
\hline & Delay & GRP $x$ & - & & & & & 0.027 \\
\hline & & S1BC & 0.479 & & & & & \\
\hline \multirow[t]{10}{*}{ Females } & HVLT-R & GRP & - & 0.691 & - & - & $0.025 *$ & 0.347 \\
\hline & Immediate & & 1.785 & & 0.794 & 2.582 & & \\
\hline & Recall & S1BC & - & & & & & 0.004 \\
\hline & & & 0.187 & & & & & \\
\hline & & GRP x & - & & & & & 0.009 \\
\hline & & S1BC & 0.334 & & & & & \\
\hline & HVLT-R & GRP & - & 0.389 & - & - & $0.001 *$ & 0.612 \\
\hline & Delayed Recall & & 1.944 & & 1.054 & 5.002 & & \\
\hline & & S1BC & - & & & & & $<0.001$ \\
\hline & & & 0.016 & & & & & \\
\hline
\end{tabular}




\begin{tabular}{|c|c|c|c|c|c|c|c|}
\hline & GRP x & - & & & & & 0.023 \\
\hline & S1BC & 0.438 & & & & & \\
\hline HVLT-R & GRP & - & 0.338 & - & - & $<0.001 *$ & 0.696 \\
\hline Retention & & 1.913 & & 1.124 & 5.653 & & \\
\hline & S1BC & 0.520 & & & & & 0.057 \\
\hline & GRP $x$ & - & 0.393 & - & - & $0.026 *$ & 0.145 \\
\hline & S1BC & 1.016 & & 0.870 & 2.582 & & \\
\hline Associative & GRP & - & 0.548 & - & - & $0.049 *$ & 0.194 \\
\hline Recognition & & 1.213 & & 0.593 & 2.213 & & \\
\hline & S1BC & - & 0.522 & - & - & 0.062 & 0.170 \\
\hline & & 1.082 & & 0.806 & 2.073 & & \\
\hline & GRP x & 0.838 & & & & & 0.068 \\
\hline & S1BC & & & & & & \\
\hline Spatial & GRP & - & & & & & 0.074 \\
\hline Working & & 0.522 & & & & & \\
\hline Memory - & S1BC & 0.618 & 0.284 & 0.661 & 2.181 & 0.052 & 0.114 \\
\hline Trial 1 & GRP $x$ & - & 0.346 & - & - & $0.001 *$ & 0.468 \\
\hline & S1BC & 1.527 & & 1.562 & 4.413 & & \\
\hline Spatial Working & GRP & 0.226 & & & & & 0.009 \\
\hline Memory - Trial & S1BC & 0.074 & & & & & 0.001 \\
\hline 2 & GRP x & - & & & & & 0.084 \\
\hline & S1BC & 0.804 & & & & & \\
\hline Spatial & GRP & - & & & & & 0.013 \\
\hline Working & & 0.245 & & & & & \\
\hline Memory - & S1BC & 0.349 & & & & & 0.029 \\
\hline Delay & GRP x & - & 0.377 & - & - & $0.004 *$ & 0.289 \\
\hline & S1BC & 1.354 & & 1.228 & 3.593 & & \\
\hline
\end{tabular}

1001 Note. Male $n=16$ per model. Female $n=14-15$ per model. TSST $=$ Trier social stress test. Group $1002(G R P)=(1)$ amnestic mild cognitive impairment vs. (0) normal aging. SIC = stress-induced cortisol. $1003 D C=$ diurnal cortisol. ${ }^{*}=p<0.05$. Effects and interactions with $p \sim 1.0$ have SE, $\beta$, and $t$ values 1004 shown.

1005

1006 
1007 Table 4. Session 2 cortisol moderation models: main effects and interactions.

\begin{tabular}{|c|c|c|c|c|c|c|c|c|}
\hline & Model & Term & $B$ & $S E$ & $\beta$ & $t$ & $P$ & $s r^{2}$ \\
\hline \multirow[t]{45}{*}{ Males } & HVLT-R Immediate & GRP & - & 0.507 & - & - & $0.002 *$ & 0.387 \\
\hline & Recall & & 1.933 & & 0.872 & 3.816 & & \\
\hline & & S2BC & 0.154 & & & & & 0.010 \\
\hline & & GRP $x$ & - & & & & & $<0.001$ \\
\hline & & S2BC & 0.033 & & & & & \\
\hline & HVLT-R Delayed & GRP & - & 0.327 & - & - & $<0.001 *$ & 0.653 \\
\hline & Recall & & 2.376 & & 1.133 & 7.272 & & \\
\hline & & S2BC & - & & & & & $<0.001$ \\
\hline & & & 0.030 & & & & & \\
\hline & & GRP $x$ & 0.597 & 0.301 & 0.368 & 1.984 & 0.071 & 0.049 \\
\hline & & S2BC & & & & & & \\
\hline & HVLT-R Retention & GRP & - & 0.579 & - & - & 0.017* & 0.317 \\
\hline & & & 1.597 & & 0.789 & 2.757 & & \\
\hline & & S2BC & - & & & & & $<0.001$ \\
\hline & & & 0.020 & & & & & \\
\hline & & GRP $x$ & 0.216 & & & & & 0.007 \\
\hline & & S2BC & & & & & & \\
\hline & Associative & GRP & - & 0.468 & - & - & $0.002 *$ & 0.431 \\
\hline & Recognition & & 1.890 & & 0.921 & 4.035 & & \\
\hline & & S2BC & - & & & & & 0.001 \\
\hline & & & 0.051 & & & & & \\
\hline & & GRP $x$ & 0.286 & & & & & 0.012 \\
\hline & & S2BC & & & & & & \\
\hline & Spatial Working & GRP & 0.589 & & & & & 0.060 \\
\hline & Memory - Trial 1 & S2BC & 0.051 & & & & & 0.002 \\
\hline & & GRP $x$ & 0.438 & & & & & 0.039 \\
\hline & & S2BC & & & & & & \\
\hline & Spatial Working & GRP & 1.509 & 0.723 & 0.700 & 2.086 & 0.061 & 0.250 \\
\hline & Memory - Trial 2 & S2BC & & & & & & 0.002 \\
\hline & & & 0.064 & & & & & \\
\hline & & GRP $x$ & - & & & & & 0.004 \\
\hline & & S2BC & 0.176 & & & & & \\
\hline & Spatial Working & GRP & 1.366 & & & & & 0.193 \\
\hline & Memory - Delay & S2BC & - & & & & & 0.016 \\
\hline & & & 0.196 & & & & & \\
\hline & & GRP x & - & & & & & 0.001 \\
\hline & & S2BC & 0.082 & & & & & \\
\hline & TSST Speech Task & GRP & - & & & & & 0.049 \\
\hline & Anxiety & & 0.541 & & & & & \\
\hline & & S2BC & 0.740 & 0.212 & 0.831 & 3.494 & 0.004* & 0.366 \\
\hline & & GRP x & - & 0.389 & - & - & 0.014* & 0.248 \\
\hline & & S2BC & 1.118 & & 0.832 & 2.876 & & \\
\hline & TSST Counting Task & GRP & 0.006 & & & & & $<0.001$ \\
\hline & Anxiety & S2BC & - & & & & & $<0.001$ \\
\hline & & & 0.010 & & & & & \\
\hline
\end{tabular}




\begin{tabular}{|c|c|c|c|c|c|c|c|c|}
\hline & & $\begin{array}{l}\text { GRP x } \\
\text { S2BC }\end{array}$ & 0.673 & & & & & 0.073 \\
\hline \multirow[t]{44}{*}{ Females } & HVLT-R Immediate & GRP & - & 0.373 & - & - & $0.025 *$ & 0.207 \\
\hline & Recall & & 0.966 & & 0.611 & 2.589 & & \\
\hline & & S2BC & - & & & & & 0.003 \\
\hline & & & 0.101 & & & & & \\
\hline & & GRP $x$ & 0.649 & & & & & 0.070 \\
\hline & & S2BC & & & & & & \\
\hline & HVLT-R Delayed & GRP & - & 0.594 & - & - & 0.041* & 0.307 \\
\hline & Recall & & 1.390 & & 0.745 & 2.341 & & \\
\hline & & S2BC & 0.232 & & & & & 0.011 \\
\hline & & GRP $x$ & - & & & & & 0.006 \\
\hline & & S2BC & 0.232 & & & & & \\
\hline & HVLT-R Retention & GRP & - & 0.645 & - & - & 0.067 & 0.251 \\
\hline & & & 1.311 & & 0.673 & 2.034 & & \\
\hline & & S2BC & 0.285 & & & & & 0.015 \\
\hline & & GRP x & - & & & & & 0.049 \\
\hline & & S2BC & 0.666 & & & & & \\
\hline & Associative & GRP & - & & & & & 0.067 \\
\hline & Recognition & & 0.672 & & & & & \\
\hline & & S2BC & - & & & & & 0.010 \\
\hline & & & 0.237 & & & & & \\
\hline & & GRP $x$ & - & & & & & 0.001 \\
\hline & & S2BC & 0.082 & & & & & \\
\hline & Spatial Working & GRP & 0.393 & & & & & 0.017 \\
\hline & Memory - Trial 1 & S2BC & 0.173 & & & & & 0.004 \\
\hline & & GRP $x$ & 0.267 & & & & & 0.006 \\
\hline & & S2BC & & & & & & \\
\hline & Spatial Working & GRP & 0.645 & & & & & 0.069 \\
\hline & Memory - Trial 2 & S2BC & 0.027 & & & & & $<0.001$ \\
\hline & & GRP $x$ & - & & & & & 0.024 \\
\hline & & S2BC & 0.436 & & & & & \\
\hline & Spatial Working & GRP & 0.707 & & & & & 0.094 \\
\hline & Memory - Delay & S2BC & 0.442 & & & & & 0.045 \\
\hline & & GRP $x$ & - & & & & & 0.018 \\
\hline & & S2BC & 0.359 & & & & & \\
\hline & TSST Speech Task & GRP & - & 0.495 & - & - & 0.034* & 0.199 \\
\hline & Anxiety & & 1.265 & & 0.599 & 2.557 & & \\
\hline & & S2BC & 2.016 & 0.446 & 1.356 & 4.520 & $0.002 *$ & 0.622 \\
\hline & & GRP $x$ & - & 0.571 & - & - & 0.057 & 0.150 \\
\hline & & S2BC & 1.266 & & 0.632 & 2.217 & & \\
\hline & TSST Counting Task & GRP & 0.936 & & & & & 0.136 \\
\hline & Anxiety & S2BC & - & & & & & 0.003 \\
\hline & & & 0.119 & & & & & \\
\hline & & GRP $x$ & - & & & & & 0.001 \\
\hline & & S2BC & 0.081 & & & & & \\
\hline
\end{tabular}

1008 Note. Male $n=15-16$ per model. Female $n=10-15$ per model. TSST $=$ Trier social stress test. Group $1009(G R P)=(1)$ amnestic mild cognitive impairment vs. (0) normal aging. SIC = stress-induced cortisol. 
bioRxiv preprint doi: https://doi.org/10.1101/757484; this version posted July 23,2020 . The copyright holder for this preprint (which was not certified by peer review) is the author/funder, who has granted bioRxiv a license to display the preprint in perpetuity. It is made available under aCC-BY-NC-ND 4.0 International license.

$1010 D C=$ diurnal cortisol. $*=p<0.05$. Effects and interactions with $p \sim 1.0$ have SE, $\beta$, and $t$ values 1011 shown. 
Figure Captions

1013 Figure 1. Timeline of Sessions and Testing within Sessions. Exact times varied based on individual 1014 variability.

1015

1016 Figure 2. (A) $\log _{10}$ transformed salivary cortisol at two separate time points and (B) $\log _{10}$

1017 transformed area under the curve with respect to the ground (AUCg) cortisol during Session 1 in 1018 normal aging (NA; $n=9$ females, $n=7$ males) and amnestic mild cognitive impaired (aMCI; $n=6$ 1019 females, $n=9$ males) participants. (C) Log transformed salivary cortisol at four separate time points 1020 and (D) $\log _{10}$ transformed AUCg cortisol during Session 2 in NA ( $n=9$ females, $n=7$ males) and 1021 aMCI ( $n=6$ females, $n=9$ males). \# denotes a Main effect of sex, group, or time, $p<0.05$. * denotes 1022 aMCI males differ from all other groups at a single time point or compared to all other groups for 1023 AUCg cortisol, $p<0.05$. \& denotes aMCI females or males differ from NA females or males 1024 respectively at a single time point, $p<0.05$.

1025

1026 Figure 3. Hopkins Verbal Learning Test-Revised (HVLT-R) (A) immediate recall, (B) delayed recall, 1027 and (C) retention scores during session 1 and session 2 (after Trier Social Stress Test) in normal aging 1028 (NA; $n=9$ females, $n=7$ males) and amnestic mild cognitive impairment (aMCI; $n=6$ females, $n=9$ 1029 males). \# denotes Main effect of group or session, $p<0.05$. * denotes a significant increase from 1030 Session 1 and Session 2 in NA males and females, $p<0.05$. \& denotes a significant decrease from 1031 Session 1 and Session 2 in aMCI males, $p<0.05$.

1032

1033 Figure 4. (A) Face-name associative recognition and (B) spatial working memory performance during 1034 Session 1 and Session 2 in normal aging (NA; $n=9$ females, $n=7$ males) and amnestic mild cognitive 1035 impaired (aMCI; $n=6$ females, $n=9$ males) participants. \# denotes main effect of sex or group $p<$ 1036 0.05. * denotes fewer number of choices in delay trial compared to Trial 1 and Trial 2.

1038 Figure 5. (A) $\log _{10}$ transformed diurnal salivary cortisol measures at five time points averaged across 1039 three consecutive days in normal aging (NA) males $(n=7)$ and amnestic mild cognitive impairment 1040 (aMCI; $n=9$ ). (B) Log transformed area under the curve with respect to the ground (AUCg) diurnal 1041 cortisol in NA and aMCI males.

1042 Figure 6. Standardized moderation effects of Session 1 cortisol (AUCg) on the relationship between 1043 participant group (normal aging (NA), amnestic mild cognitive impairment (aMCI)) and Session 1 (A) 
1044 associative recognition scores and (B) spatial working memory task Trial 2 choices in males $(n=7$ NA, $1045 n=9 \mathrm{aMCI}$ ) and (C) Hopkins Verbal Learning Test-Revised (HVLT-R) retention scores, and (D) 1046 spatial working memory task Trial 1 and (E) delay choices in females $(n=9 \mathrm{NA}, n=6 \mathrm{aMCI})$. 1047

1048 Figure 7. Standardized moderation effects of Session 2 cortisol (AUCg) on the relationship between 1049 participant group (normal aging (NA), amnestic mild cognitive impairment (aMCI)) and Session 2 (A) 1050 perceived trier social stress test (TSST) speech anxiety ratings and (B) Hopkins Verbal Learning Test1051 Revised (HVLT-R) delayed recall scores in males ( $n=7$ NA, $n=9$ aMCI) and Session 2 (C) perceived 1052 anxiety rating for the Trier Social Stress Test in females ( $n=9 \mathrm{NA}, n=6$ aMCI). 
bioRxiv preprint doi: https://doi.org/10.1101/757484; this version posted July 23,2020 . The copyright holder for this preprint (which was not certified by peer review) is the author/funder, who has granted bioRxiv a license to display the preprint in perpetuity. It is made available under aCC-BY-NC-ND 4.0 International license.

\section{$\underline{\text { Session } 1}$}

\begin{tabular}{|c|c|}
\hline 10:00 & Consent, interview, and instructions \\
\hline 10:10 & Saliva Sample \#1 \\
\hline 10:15 & Face / Name Associative Memory Version A - Immediate \\
\hline 10:30 & Hopkins Verbal Learning Test-Revised: Form 6-Immediate \\
\hline 10:35 & Spatial Working Memory Version A - Immediate \\
\hline 10:45 & Trail Making Test - A \& B \\
\hline 10:50 & Face / Name Associative Memory Version A - Delay \\
\hline 10:58 & Hopkins Verbal Learning Test-Revised: Form 6 - Delay \\
\hline 11:00 & Mini-Mental Status Exam \\
\hline 11:05 & Spatial Working Memory Version A - Delay (20-25 minutes) \\
\hline 11:10 & WAIS-III Digit Span \\
\hline 11:20 & Boston Naming Test (split half - odds) \\
\hline 11:30 & Rey-Osterreith Figure Copy and Immediate Recall \\
\hline 11:40 & WAIS-III Vocabulary (split half - odds) \\
\hline 11:50 & Hospital Anxiety and Depression Scale \\
\hline 11:55 & Saliva Sample \#2, schedule at-home saliva collection, book session 2 \\
\hline $12: 05$ & Session ends \\
\hline
\end{tabular}

\section{Session 2}

\begin{tabular}{l}
$10: 00$ \\
$10: 10$ \\
$10: 13$ \\
$10: 18$ \\
$10: 28$ \\
$10: 31$ \\
\hline $10: 43$ \\
$10: 48$ \\
\hline $11: 03$ \\
$11: 06$ \\
\hline $11: 16$ \\
\hline $11: 18$ \\
\hline $11: 23$ \\
\hline $11: 28$ \\
\hline $11: 33$ \\
\hline $11: 43$ \\
\hline $11: 44$
\end{tabular}

\begin{tabular}{|c|}
\hline Beck's Anxiety and Depression Inventories \\
\hline Saliva sample \#1 ( 20 minutes before TSST) \\
\hline Instructions and consent for Trier Social Stress Test \\
\hline Trier Social Stress Test - preparation of speech \\
\hline Saliva sample \#2 (immediately before TSST Speech) \\
\hline Trier Social Stress Test \\
\hline Hopkins Verbal Learning Test-Revised: Form 5 - Immediate \\
\hline Face / Name Associative Memory Version B - Immediate \\
\hline Saliva Sample \#3 ( 30 minutes after beginning of TSST) \\
\hline Spatial Working Memory Version B - Immediate \\
\hline Hopkins Verbal Learning Test-Revised: Form 5 - Delay \\
\hline Face / Name Associative Memory Version B - Delay \\
\hline Spatial Working Memory Version B - Delay \\
\hline Trail Making Test - A \\
\hline Coping Strategies Scale, SF-36 Health Survey \\
\hline Saliva Sample \#4 ( 60 minutes after beginning of TSST) \\
\hline Session ends \\
\hline
\end{tabular}



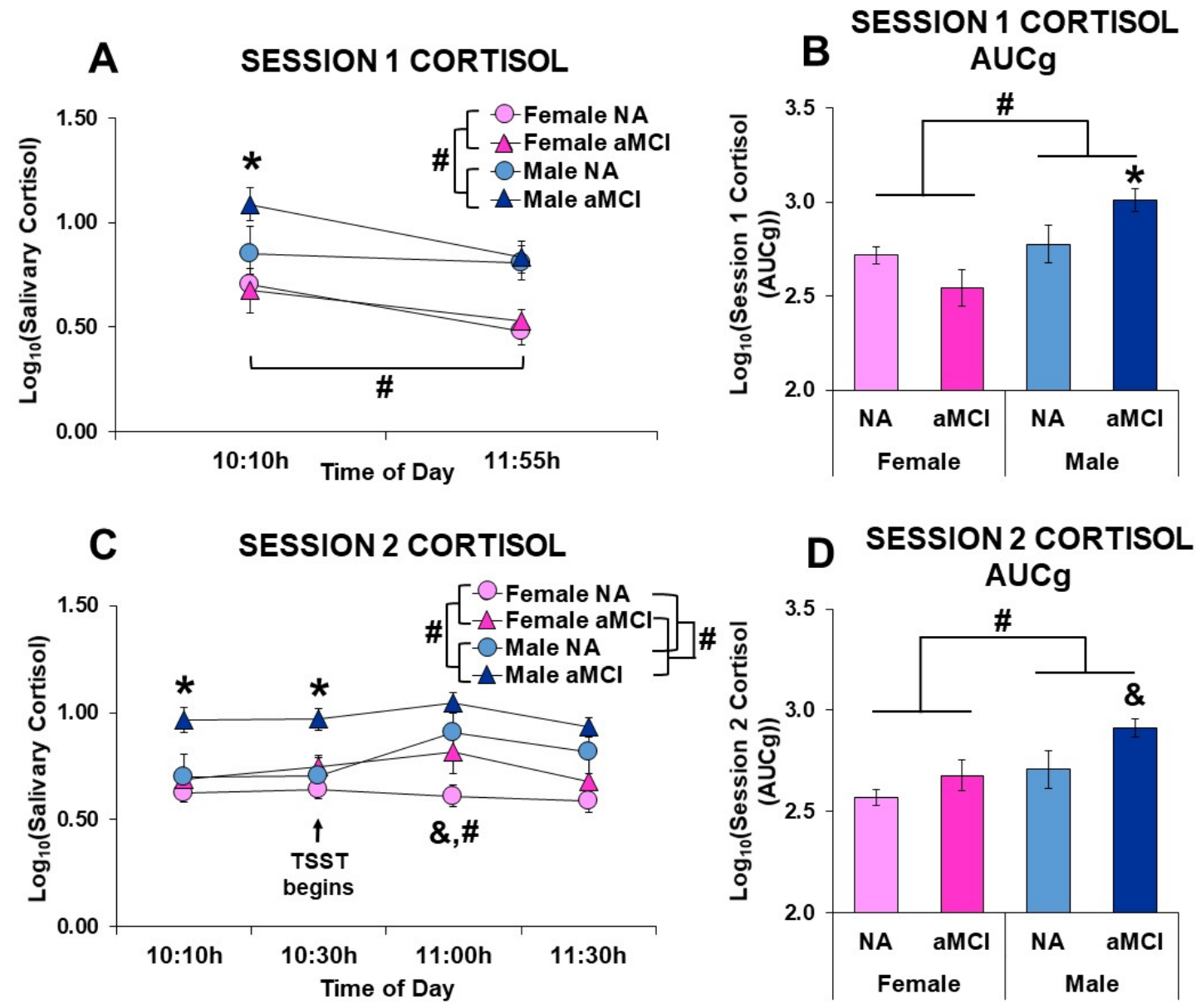


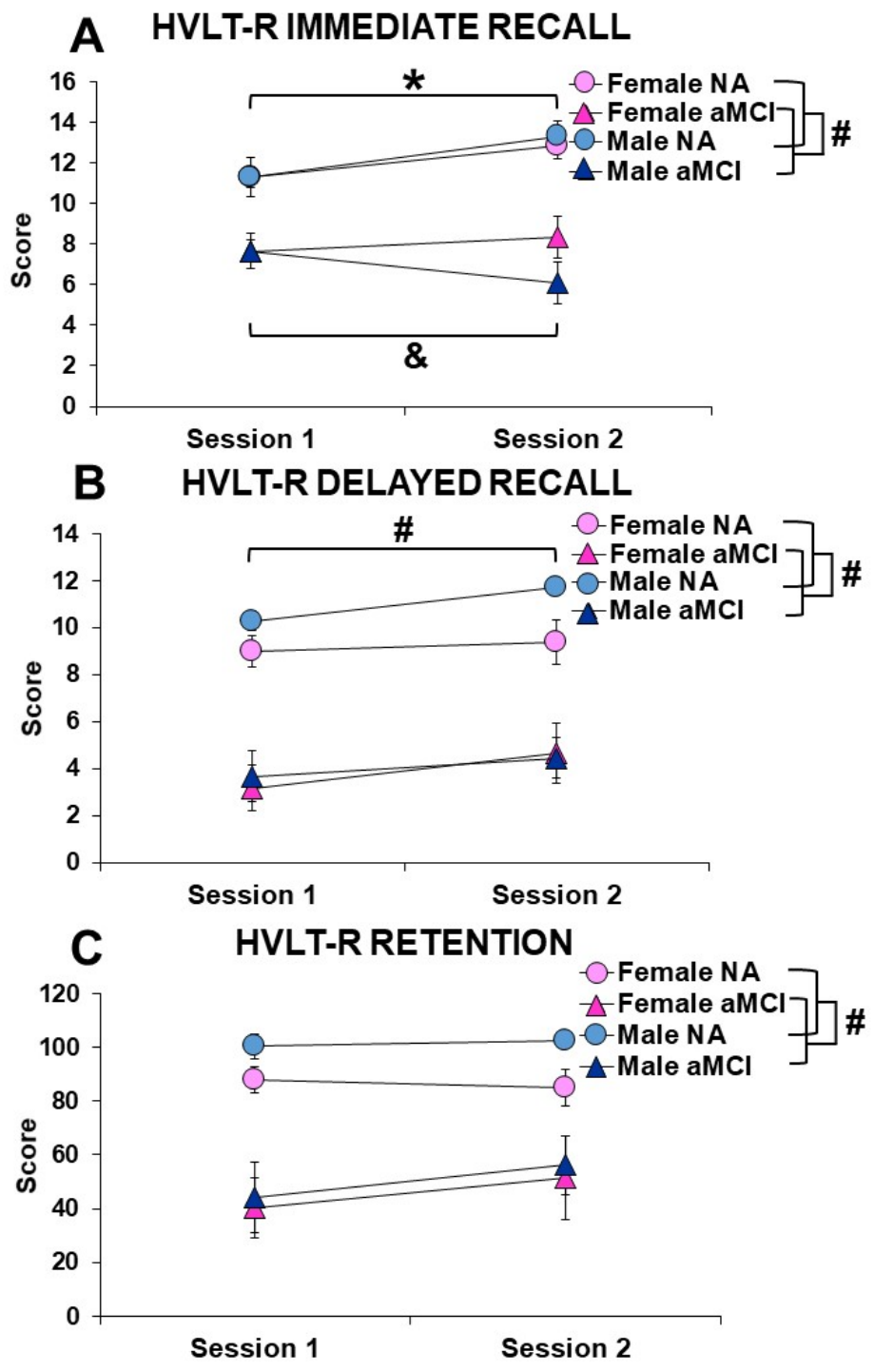




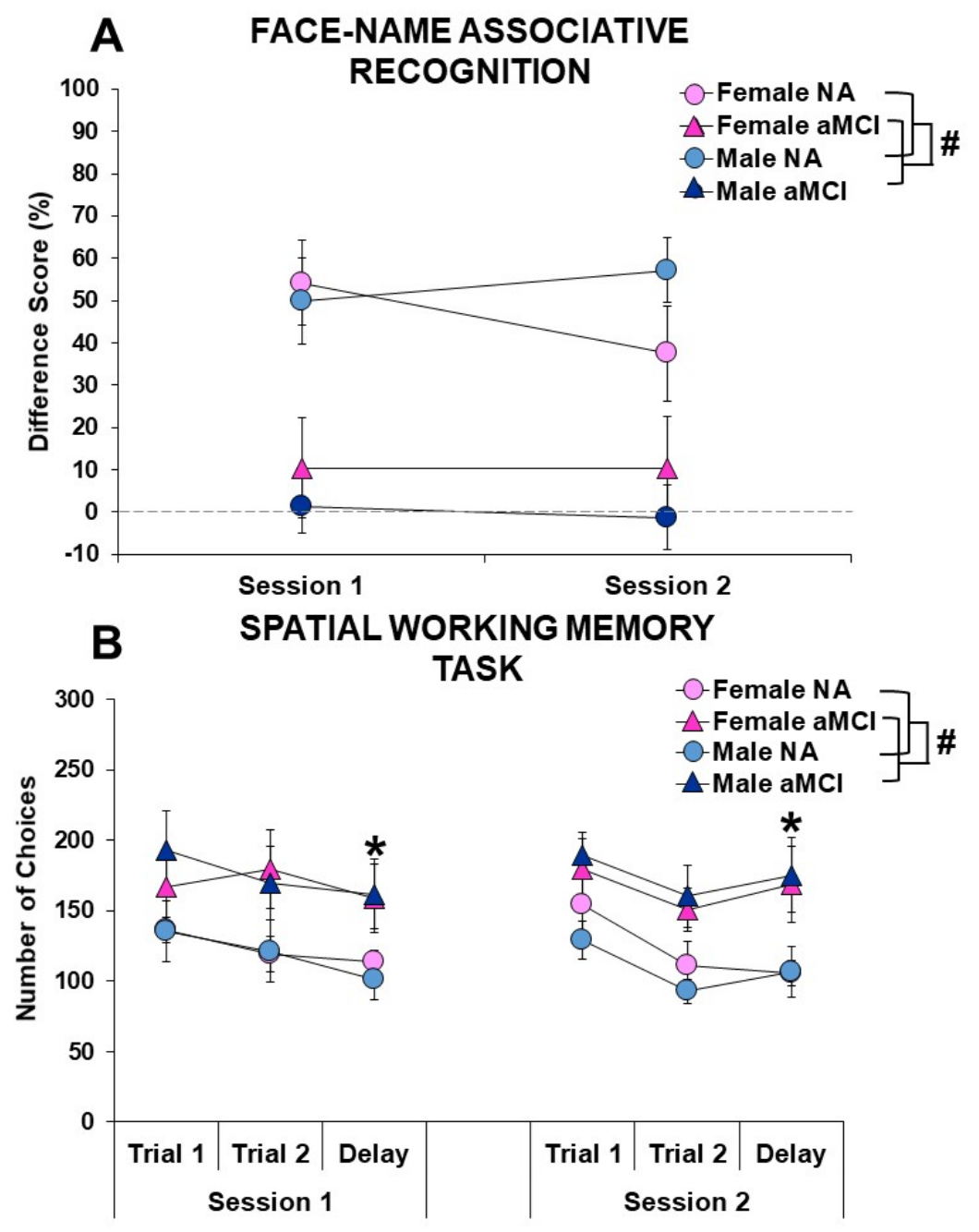




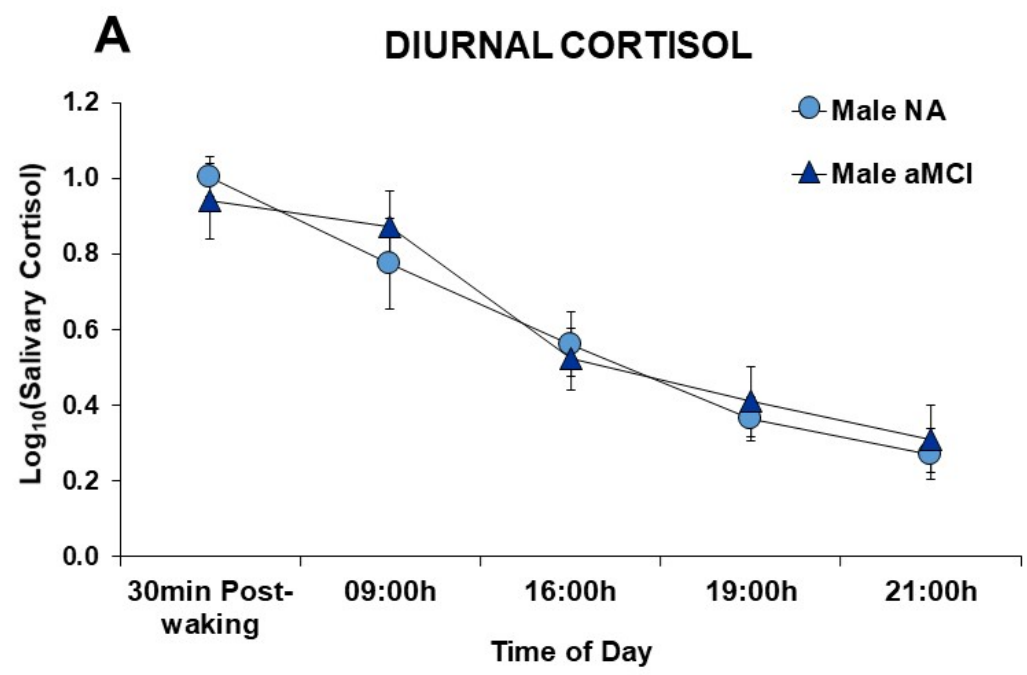

B

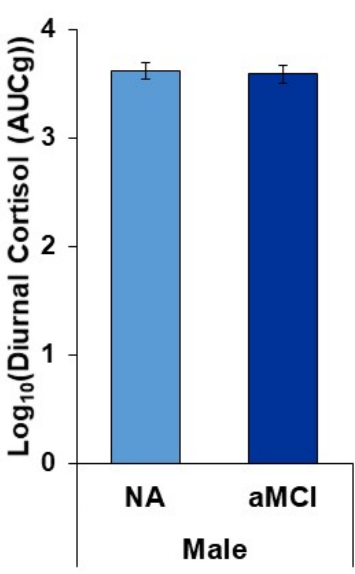


SESSION 1: MODERATION MODELS
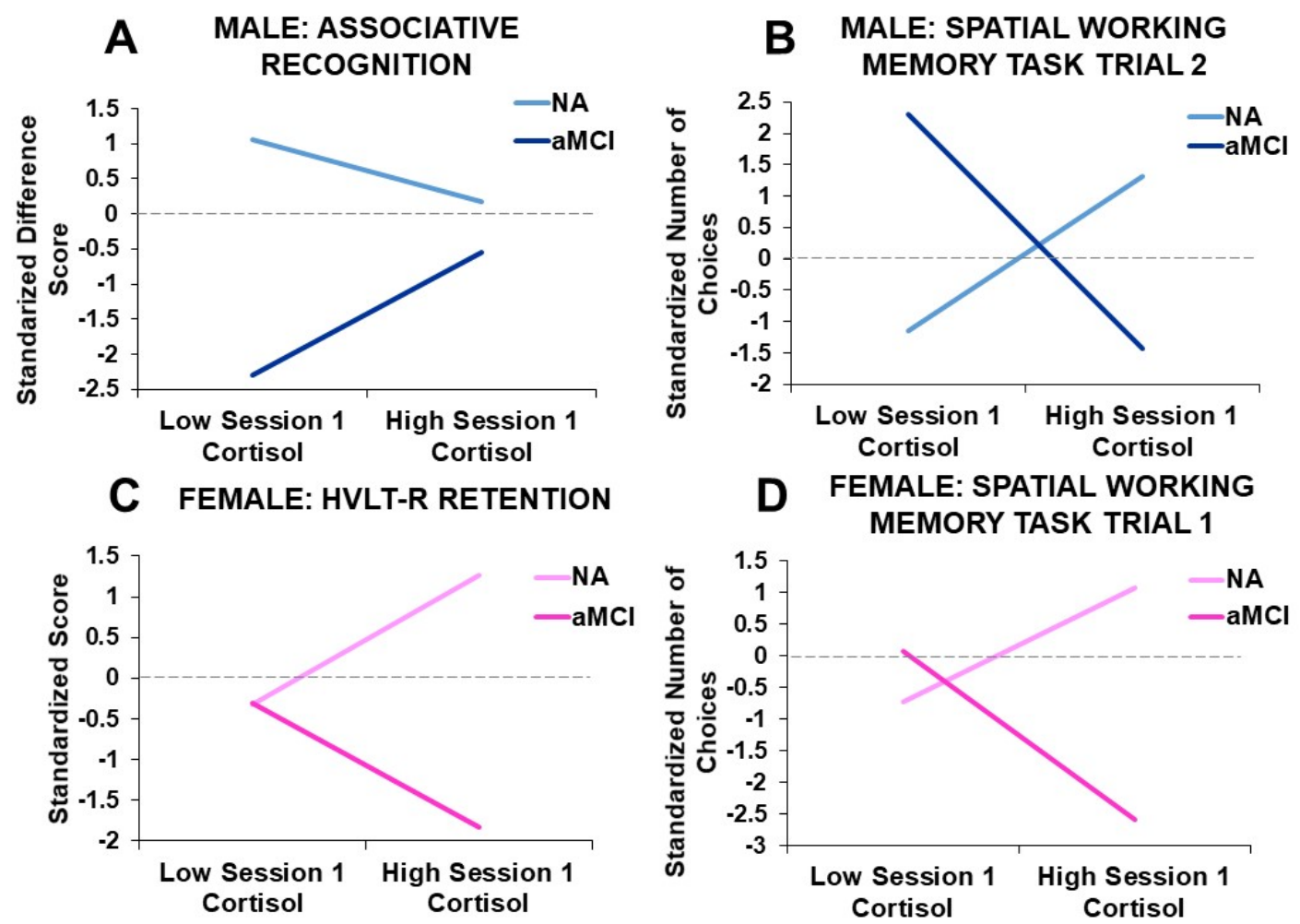

E FEMALE: SPATIAL WORKING

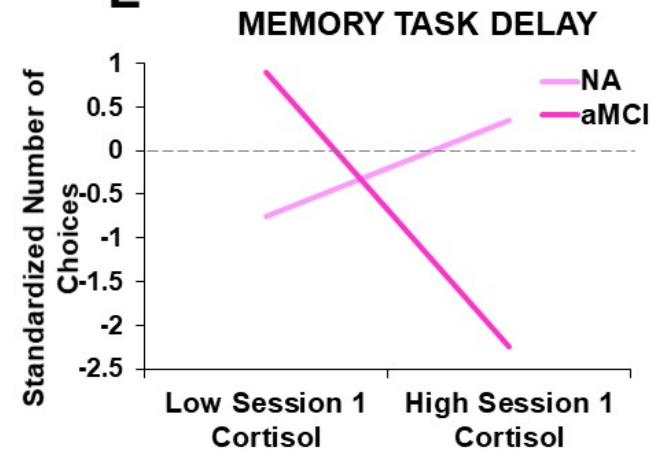




\section{SESSION 2: MODERATION MODELS}

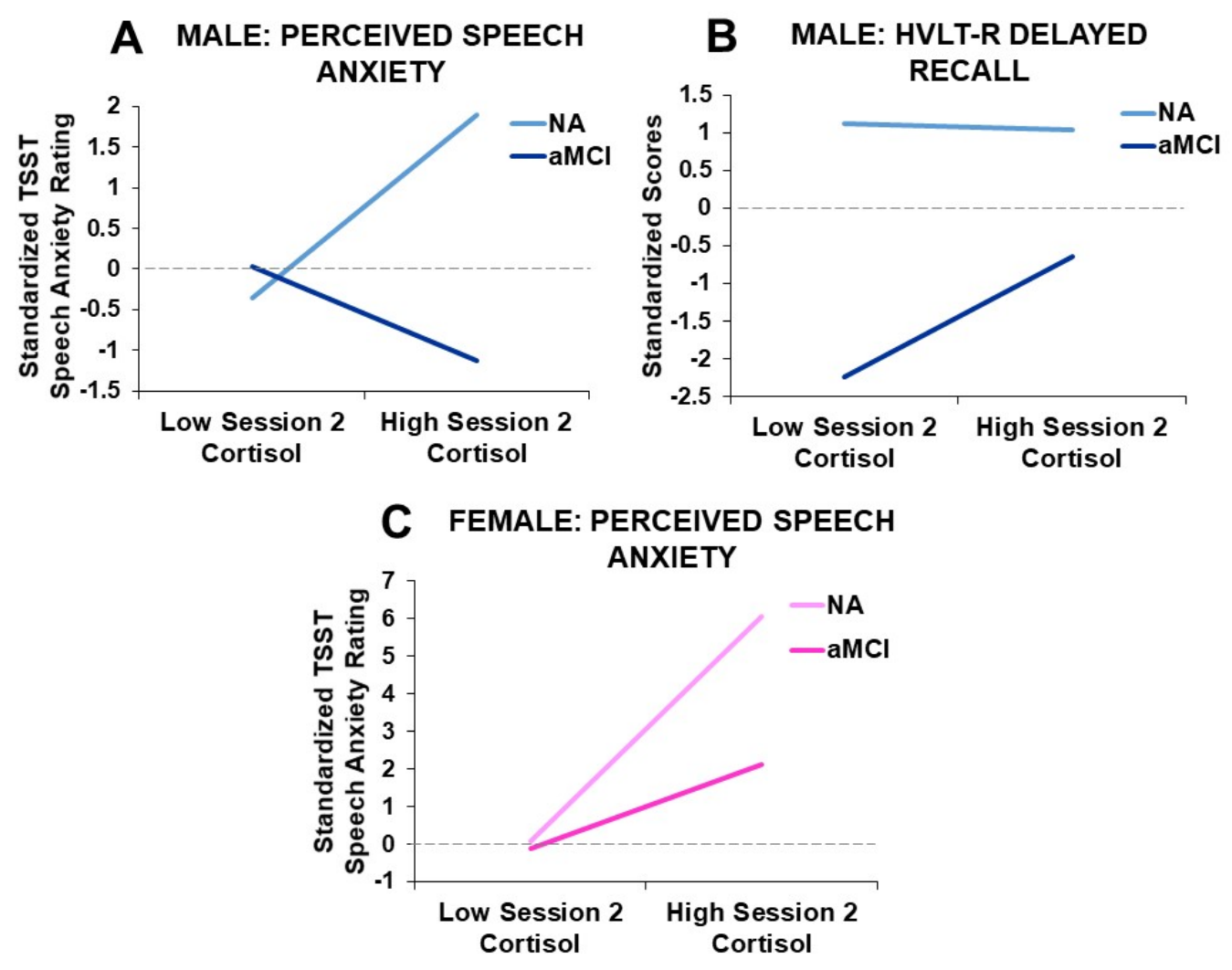

